\title{
The birds of Fazenda Brejão: a conservation priority area of Cerrado in northwestern Minas Gerais, Brazil
}

\author{
Luciene Carrara Paula Faria', Lucas Aguiar Carrara', Frederico Queiroga do Amaral ${ }^{I}$, \\ Marcelo Ferreira de Vasconcelos ${ }^{1}$, Mauro Guimarães Diniz ${ }^{2}$, Christiane Duarte Encarnaçãó2, \\ Diego Hoffmann ${ }^{1}$, Henrique Belfort Gomes ${ }^{1}$, Leonardo Esteves Lopes $^{1}$ \& Marcos Rodrigues $^{1,3}$ \\ ${ }^{1}$ Laboratório de Ornitologia, Departamento de Zoologia, Instituto de Ciências, Biológicas - ICB, \\ Universidade Federal de Minas Gerais - UFMG, CP 486, CEP 31270-901, Belo Horizonte, MG, Brazil \\ ${ }^{2}$ Instituto Brasileiro do Meio Ambiente - IBAMA/SUPES-MG, \\ Av. Contorno, 8121, CEP 30110-051, Belo Horizonte, MG, Brazil \\ ${ }^{3}$ Corresponding author: Marcos Rodrigues,e-mail: ornito@mono.icb.ufmg.br
}

FARIA, L.C.P., CARRARA, L.A., AMARAL, F.Q., VASCONCELOS, M.F., DINIZ, M.G., ENCARNAÇÃO, C.D., HOFFMANN, D., GOMES, H.B., LOPES, L.E. \& RODRIGUES, M. The birds of Fazenda Brejão: a conservation priority area of Cerrado in northwestern Minas Gerais, Brazil. Biota Neotrop. 9(3): http:// www.biotaneotropica.org.br/v9n3/en/abstract?inventory+bn01109032009.

\begin{abstract}
The Cerrado is now considered the most threatened biome of South America. Northwestern Minas Gerais state, in southeastern Brazil, still harbors large areas of extreme importance for biological conservation of the Cerrado. The current study provides the first thorough assessment of the avifauna of a large remnant of Cerrado vegetation in this region, the Fazenda Brejão. Data are based on a bird survey conducted from November 1998 to January 2007. This area is a private property of 20.000 ha preserved that lies in a region recognized for its palm groves, a pristine Cerrado (savannah-like vegetation) and gallery forests. Bird censuses were carried out through random transects censuses, mist-net captures and recording bird vocalizations. It was recorded 273 species, belonging to 56 families. This represents $32 \%$ of all 857 bird species recorded for the Cerrado. Palm groves held approximately $42 \%$ of all recorded species. We also recorded endemic species of Cerrado as well as adjacent biomes such as the Atlantic Forest and the Caatinga. Regional threatened species were also recorded such as the Greater Rhea Rhea americana, the Chestnut-bellied Guan Penelope ochrogaster, the Bare-faced Curassow Crax fasciolata, the Black-and-white Hawk-eagle Spizastur melanoleucus, the Blue-and-yellow Macaw Ara ararauna, the Yellow-faced Parrot Alipiopsitta xanthops and the Minas Gerais Tyrannulet Phylloscartes roquettei. This species diversity reveals the biological importance of the region. The establishment of a natural reserve in the region will be an essential measure to guarantee the biological conservation of one of the best preserved areas of Cerrado to date in southeastern Brazil.
\end{abstract}

Keywords: avian inventory, biodiversity, Cerrado, São Francisco river.

FARIA, L.C.P., CARRARA, L.A., AMARAL, F.Q., VASCONCELOS, M.F., DINIZ, M.G., ENCARNAÇÃO, C.D., HOFFMANN, D., GOMES, H.B., LOPES, L.E. \& RODRIGUES, M. Aves da Fazenda Brejão: uma área prioritária para conservação do Cerrado no noroeste de Minas Gerais, Brasil. Biota Neotrop. 9(3): http:// www.biotaneotropica.org.br/v9n3/pt/abstract?inventory+bn01109032009.

Resumo: O Cerrado é considerado um dos biomas mais ameaçados da América do Sul. O noroeste do estado de Minas Gerais, sudeste do Brasil, ainda possui grandes áreas de importância biológica extrema para a conservação do Cerrado. Este estudo apresenta o primeiro levantamento sistemático da avifauna de uma grande área remanescente de Cerrado nesta região, a Fazenda Brejão. Os dados são baseados em levantamentos conduzidos de novembro de 1998 a janeiro de 2007. A área é uma propriedade privada de mais de 20.000 ha preservados que se situa numa região conhecida por suas veredas de buritizais, Cerrado e matas ciliares preservados. O censo de aves foi feito em transeções aleatórias, captura e marcação de indivíduos e gravação de vocalizações. Foram registradas 273 espécies de 56 famílias. O total representa 32\% das 857 espécies já registradas no Cerrado. As veredas de buritizais contêm aproximadamente $42 \%$ de todas as espécies registradas. Também foram registradas espécies localmente ameaçadas, como a ema Rhea americana, o jacu-de-barriga-castanha Penelope ochrogaster, o mutum-de-penacho Crax fasciolata, o gavião-pato Spizastur melanoleucus, a arara-canindé Ara ararauna, o papagaio-galego Alipiopsitta xanthops, e o cara-dourada Phylloscartes roquettei. Essa diversidade de espécies revela a importância biológica da região. A consolidação de unidades de conservação na região será medida essencial para garantir a conservação da biodiversidade de umas das áreas mais bem preservadas do Cerrado até o momento no sudeste do Brasil.

Palavras-chave: inventário de aves, biodiversidade, Cerrado, rio São Francisco. 


\section{Introduction}

The Cerrado biome encompasses more than 1.8 million $\mathrm{km}^{2}$ in Central Brazil, northeastern Paraguay and eastern Bolivia (Ab'Saber 1977). Most of the region is covered by savannah-like vegetation locally known as 'Cerrado' [see (Eiten 1972) for a detailed description of the vegetation] and holds an extremely rich diversity of organisms with high levels of plant and animal endemism (Oliveira \& Marquis 2002). The Cerrado is now considered the most threatened biome of South America (Oliveira \& Marquis 2002). Nevertheless, its high diversity is not distributed uniformly across the region because the biome is made of a complex mosaic of xeric (dry open grasslands to open and dense scrublands) and mesic habitats (evergreen gallery forests, palm groves, and semi-deciduous to deciduous dry forest patches growing in rich-soil areas (Oliveira Filho \& Ratter 2002). Such a diversity of environmental conditions may result in a variety of patterns of species diversity (Rodrigues \& Faria 2007).

Despite its importance, the Cerrado region holds the lowest number of protected areas in Brazil (Machado et al. 2004a, Olmos 2006), and the protected areas established at present do not embrace its habitat diversity (Drummond et al. 2005).

Northwestern Minas Gerais state, in southeastern Brazil, still harbors large areas of extreme importance for biological conservation (Drummond et al. 2005). The region still holds populations of rare and threatened mammals such as the Marsh Deer Blastocerus dichotomus, peccaries and other large species (Vieira et al. 2005). Also, numerous threatened birds occur in the area such as the Yellow-faced Parrot Alipiopsitta xanthops (Carrara et al. 2007) and one of the few populations of the Chestnut-bellied Guan Penelope ochrogaster along the São Francisco River (Collar et al. 1992, Olmos 1998, Antas 2006). The Cerrado is now regarded as the last frontier for agricultural enterprises in Brazil, mainly for soybeans (Machado et al. 2004b, Marris 2005).

Bird inventories are still scarce in Cerrado (Silva 1995a, Silva \& Santos 2005, Bagno 1998, Silveira 1998, Rodrigues et al. 2005) and it is paramount to increase our knowledge of the distribution of the endemic species of this biome.

From 1991 to 1994, two of us (CDE and MGD) carried out a bird survey at Fazenda Brejão, a remnant area of more than 20,000 ha of pristine Cerrado vegetation in northwestern Minas Gerais state. They recorded 223 bird species (unpublished data), and this work was instrumental in including the area as a waterbird monitoring area (Wetlands for the Americas 1993).

The current study provides the first thorough assessment of the avifauna of Fazenda Brejão across a complex of vegetation types, including the palm grove swamps, with information on their seasonality and frequency of occurrence.

\section{Material and Methods}

Fazenda Brejão (FB) is a 32,000 ha private area at the municipality of Brasilândia de Minas ( $17^{\circ} 01^{\prime} 48^{\prime \prime} \mathrm{S}$ and $45^{\circ}$ 54' 04" W at $550 \mathrm{~m}$ above sea level) in northwestern state of Minas Gerais, southeastern Brazil. The region, originally dominated by woodlands and palm groves at present suffers from deforestation initiated in the early 1960's by the construction of the political capital of Brazil, Brasília. About 10,000 ha of FB holds a commercial Eucalyptus plantation, with the remaining 21,747 ha being of undisturbed vegetation characterized by a woodland (5-8 $\mathrm{m}$ tall) with closed scrub and scattered trees known as 'Cerrado stricto sensu', crossed by patches of palm groves, and gallery forests along the Paracatu River (a major tributary of middle São Francisco River basin). Seasonal flooded areas occur along the plane of the Paracatu River. These areas, as well as the palm groves swamp account for the name of the site: Fazenda Brejão, which in Portuguese means 'large swampy farm'. Total annual precipitation is around 1,200 mm, but much variation can occur from year to year. Most of the rain (80\%) falls from November to March, while there is virtually no rain between May and August. Therefore, the region is characterized by strong seasonality, and only two seasons can be distinguished: the rainy and dry seasons (Carrara et al. 2007).

Field work was carried out from November 1998 to January 2007 , for a total of 30 visits to FB of five to seven days each. We conducted random transects, point count and mist-netting surveys in the main vegetation types of FB. Birds were identified with the aid of binoculars, and tape recordings. Recordings of the bird voices were made using a Sony TCM-5000 tape-recorder and a Sennheiser ME-66 directional microphone. Some voucher specimens were collected and are housed at the bird collection of the 'Departamento de Zoologia' of 'Universidade Federal de Minas Gerais' (DZUFMG). Blood samples of many specimens have been deposited in the 'Banco de DNA do Laboratório de Biologia e Evolução Molecular' of the 'Departamento de Biologia Geral of Universidade Federal de Minas Gerais' (Santos et al. 2002).

Each mist netted bird received a numbered metal ring from the Brazilian Environmental Agency (IBAMA/CEMAVE license n. 12743-1) and was subsequently released. Brood patch and molt were recorded for each captured bird.

For each species was assigned an abundance class according to its frequency of occurrence following Hilty \& Brown (1986) and Rodrigues et al. (2005). The frequency of occurrence for each species was calculated as the number of surveys in which a given species was detected in any of the methods: A, (common) recorded 75-100\% of the visits; C, (fairly common) 50-74 \%; E, (uncommon) $25-49 \%$; R, (rare) recorded on less than $25 \%$ of the visits; $\mathrm{O}$, (occasional) wanderers or vagrants recorded without any clear pattern in occurrence.

Seasonal patterns are based on our original data, but to understand global migration patterns we consulted several key references (Ridgely \& Tudor 1989, 1994, Sick 1993, Stotz et al. 1996).

Taxonomic order follows the Brazilian Committee for Ornithological Records (CBRO 2008). Endemic status of birds for the Cerrado follows (Silva \& Bates 2002); for the Atlantic forest we follow (Silva et al. 2004) and for the Caatinga we use Sick (1993). Conservation status at regional scale follows Machado et al. (1998) and at national scale we follow MMA (2003) and at global scale we follow BirdLife International (2000).

\section{Results}

\section{Species richness}

We recorded a total of 273 species, belonging to 56 families (Table 1). Most observed species belonged to the Order Passeriformes (140) and the family Tyrannidae (49) followed by Thraupidae (15), Trochilidae (13), and Furnariidae (12). Non passerine aquatic birds were represented by 30 species $(11 \%)$. We captured 101 species and 692 individual captures in 7,265.7 net-hours (Table 1).

Seventy species were considered common, 50 fairly common, 60 uncommon, 57 rare and 36 occasional (Table 1). Fifty-seven percent of the species were recorded throughout the year (127 every month and 29 species recorded on intercalated months). The remaining species occurred in a specific season or without any apparent pattern. We recorded reproductive activity (brood patch or nesting) for 51 species (Table 1).

Ten species are threatened in the State of Minas Gerais, and two species are threatened in Brazil. Nine species are endemic to the Cerrado, one to the Atlantic forest and three to the Caatinga dry lands of northwestern Brazil (Table 1). 
Table 1. Bird species of Fazenda Brejão, southeastern Brazil, with information on habitat associations, seasonality, annual occurrence, and conservation status. Taxonomy follows CBRO (2008). C \& E (Conservations status and endemism): ABR: threatened in Brazil; AMG: threatened in Minas Gerais state; ECe: cerrado endemic; ECa: Caatinga endemic; EMa: Atlântic Forest endemic. A (Relative abundance): A, (common) recorded 75-100\% of the visits; C, (fairly common) $50-74 \%$; E, (uncommon) $25-49 \%$; R, (rare) recorded on less than $25 \%$ of the visits; O, (occasional) wanderers or vagrants recorded without any clear pattern.

Tabela 1. Espécies de aves da Fazenda Brejão, sudeste do Brasil, com informações sobre o habitat, sazonalidade, ocorrência anual e status de conservação. Taxonomia segue CBRO (2008). C \& E (Status de conservação e endemismo): ABR: ameaçado no Brasil; AMG: ameaçado em Minas Gerais; ECe: endêmico do Cerrado; ECa: endêmico da Caatinga; EMa: endêmico da Mata Atlântica. A (Abundância relativa): A, (comum) registrado 75-100\% do total de visitas; C, (bem comum) 50\&\#8209;74\%; E, (incomum) 25-49\%; R, (raro) registrado em menos de 25\% das visitas; O, (ocasional) registrados sem nenhum padrão claro.

\begin{tabular}{|c|c|c|c|c|c|c|c|}
\hline Families and species & $\begin{array}{l}\text { Common } \\
\text { names }\end{array}$ & $\begin{array}{c}\text { Conservation } \\
\text { and endemism }\end{array}$ & Abundance & Habitat & $\begin{array}{l}\text { Month of } \\
\text { record }\end{array}$ & Reproduction & $\begin{array}{c}\text { Number } \\
\text { captured } \\
\text { birds }\end{array}$ \\
\hline \multicolumn{8}{|l|}{ RHEIDAE (1) } \\
\hline $\begin{array}{l}\text { Rhea americana } \\
\text { (Linnaeus, 1758) }\end{array}$ & Greater Rhea & AMG & $\mathrm{C}$ & $\mathrm{CH}$ & jan-dec & oct-dec & - \\
\hline \multicolumn{8}{|l|}{ TINAMIDAE (5) } \\
\hline $\begin{array}{l}\text { Crypturellus undulatus } \\
\text { (Temminck, 1815) }\end{array}$ & $\begin{array}{l}\text { Undulated } \\
\text { Tinamou }\end{array}$ & - & $\mathrm{C}$ & M C V & jan-dec & - & - \\
\hline $\begin{array}{l}\text { Crypturellus parvirostris } \\
\text { (Wagler, 1827) }\end{array}$ & $\begin{array}{l}\text { Small-billed } \\
\text { Tinamou }\end{array}$ & - & A & M C & jan-dec & - & 1 \\
\hline $\begin{array}{l}\text { Crypturellus tataupa } \\
\text { (Temminck, 1815) }\end{array}$ & Tataupa Tinamou & - & A & M C & jan-dec & - & - \\
\hline $\begin{array}{l}\text { Rhynchotus rufescens } \\
\text { (Temminck, 1815) }\end{array}$ & $\begin{array}{l}\text { Red-winged } \\
\text { Tinamou }\end{array}$ & - & $\mathrm{E}$ & $\mathrm{CH}$ & apr-dec & - & - \\
\hline $\begin{array}{l}\text { Nothura maculosa } \\
\text { (Temminck, 1815) }\end{array}$ & Spotted Nothura & - & $\mathrm{E}$ & $\mathrm{CH}$ & jan-dec & - & - \\
\hline \multicolumn{8}{|l|}{ ANHIAMIDAE (1) } \\
\hline $\begin{array}{l}\text { Anhima cornuta } \\
\text { (Linnaeus, 1766) }\end{array}$ & Horned Screamer & - & $\mathrm{E}$ & M C V A & jan-dec & - & - \\
\hline \multicolumn{8}{|l|}{ ANATIDAE (4) } \\
\hline $\begin{array}{l}\text { Dendrocygna viduata } \\
\text { (Linnaeus, 1766) }\end{array}$ & $\begin{array}{c}\text { White-faced } \\
\text { Whistling-Duck }\end{array}$ & - & $\mathrm{E}$ & A & dec-jul & - & - \\
\hline $\begin{array}{l}\text { Dendrocygna autumnalis } \\
\text { (Linnaeus, 1758) }\end{array}$ & $\begin{array}{l}\text { Black-bellied } \\
\text { Whistling-Duck }\end{array}$ & - & $\mathrm{E}$ & A & nov-may & - & - \\
\hline $\begin{array}{l}\text { Cairina moschata } \\
\text { (Linnaeus, 1758) }\end{array}$ & Muscovy Duck & - & $\mathrm{E}$ & A & jul-apr & - & - \\
\hline $\begin{array}{l}\text { Amazonetta brasiliensis } \\
\text { (Gmelin, 1789) }\end{array}$ & Brazilian Duck & - & $\mathrm{C}$ & A & jan-dec & - & - \\
\hline \multicolumn{8}{|l|}{ CRACIDAE (3) } \\
\hline $\begin{array}{l}\text { Penelope superciliaris } \\
\text { Temminck, } 1815\end{array}$ & $\begin{array}{l}\text { Rusty-margined } \\
\text { Guan }\end{array}$ & - & $\mathrm{E}$ & M C & jan-dec & - & - \\
\hline $\begin{array}{l}\text { Penelope ochrogaster } \\
\text { Pelzeln, } 1870\end{array}$ & $\begin{array}{c}\text { Chestnut-bellied } \\
\text { Guan }\end{array}$ & $\begin{array}{l}\mathrm{ABR}, \mathrm{AMG} \\
\mathrm{ECe}\end{array}$ & $\mathrm{R}$ & M & feb,dec & - & - \\
\hline $\begin{array}{l}\text { Crax fasciolata } \\
\text { Spix, } 1825\end{array}$ & $\begin{array}{l}\text { Bare-faced } \\
\text { Curassow }\end{array}$ & AMG & $\mathrm{R}$ & M & apr,oct & - & - \\
\hline \multicolumn{8}{|l|}{ PHALACROCORACIDAE (1) } \\
\hline $\begin{array}{l}\text { Phalacrocorax brasilianus } \\
\text { (Gmelin, 1789) }\end{array}$ & $\begin{array}{l}\text { Olivaceous } \\
\text { Cormorant }\end{array}$ & - & $\mathrm{R}$ & A & jan-dec & - & - \\
\hline \multicolumn{8}{|l|}{ ANHINGIDAE (1) } \\
\hline $\begin{array}{l}\text { Anhinga anhinga } \\
\text { (Linnaeus, 1766) }\end{array}$ & Anhinga & - & $\mathrm{R}$ & A & apr-dec & - & - \\
\hline \multicolumn{8}{|l|}{ ARDEIDAE (9) } \\
\hline $\begin{array}{l}\text { Tigrisoma lineatum } \\
\text { (Boddaert, 1783) }\end{array}$ & $\begin{array}{l}\text { Rufescent } \\
\text { Tiger-Heron }\end{array}$ & - & $\mathrm{R}$ & M V & $\begin{array}{l}\text { oct,dec, } \\
\text { feb }\end{array}$ & - & - \\
\hline $\begin{array}{l}\text { Nycticorax nycticorax } \\
\text { (Linnaeus, 1758) }\end{array}$ & $\begin{array}{l}\text { Black-crowned } \\
\text { Night-Heron }\end{array}$ & - & $\mathrm{O}$ & M V & jul & - & - \\
\hline
\end{tabular}


Table 1. Continued...

\begin{tabular}{|c|c|c|c|c|c|c|c|}
\hline Families and species & $\begin{array}{c}\text { Common } \\
\text { names }\end{array}$ & $\begin{array}{c}\text { Conservation } \\
\text { and endemism }\end{array}$ & Abundance & Habitat & $\begin{array}{l}\text { Month of } \\
\text { record }\end{array}$ & Reproduction & $\begin{array}{c}\text { Number } \\
\text { captured } \\
\text { birds }\end{array}$ \\
\hline $\begin{array}{l}\text { Butorides striata } \\
\text { (Linnaeus, 1758) }\end{array}$ & Striated Heron & - & $\mathrm{E}$ & M V & feb,may & - & - \\
\hline $\begin{array}{l}\text { Bubulcus ibis } \\
\text { (Linnaeus, 1758) }\end{array}$ & Cattle Egret & - & $\mathrm{R}$ & C V A & $\begin{array}{l}\text { may,aug- } \\
\text { oct }\end{array}$ & - & - \\
\hline $\begin{array}{l}\text { Ardea cocoi } \\
\text { Linnaeus, } 1766\end{array}$ & $\begin{array}{l}\text { White-necked } \\
\text { Heron }\end{array}$ & - & $\mathrm{E}$ & A & may-aug & - & - \\
\hline $\begin{array}{l}\text { Ardea alba } \\
\text { Linnaeus, } 1758\end{array}$ & Great Egret & - & $\mathrm{C}$ & V A & jan-dec & - & - \\
\hline $\begin{array}{l}\text { Syrigma sibilatrix } \\
\text { (Temminck, 1824) }\end{array}$ & Whistling Heron & - & A & $\mathrm{CAH}$ & jan-dec & - & - \\
\hline $\begin{array}{l}\text { Pilherodius pileatus } \\
\text { (Boddaert, 1783) }\end{array}$ & Capped Heron & - & $\mathrm{R}$ & M V A & may-aug & - & - \\
\hline $\begin{array}{l}\text { Egretta thula } \\
\text { (Molina, 1782) }\end{array}$ & Snowy Egret & - & $\mathrm{C}$ & V A & jan-dec & - & - \\
\hline \multicolumn{8}{|l|}{ THRESKIORNITHIDAE (3) } \\
\hline $\begin{array}{l}\text { Mesembrinibis cayennensis } \\
\text { (Gmelin, 1789) }\end{array}$ & Green Ibis & - & $\mathrm{E}$ & M V A & jan-dec & - & - \\
\hline $\begin{array}{l}\text { Phimosus infuscatus } \\
\text { (Lichtenstein, 1823) }\end{array}$ & Bare-faced Ibis & - & $\mathrm{O}$ & A & oct & - & - \\
\hline $\begin{array}{l}\text { Theristicus caudatus } \\
\text { (Boddaert, 1783) }\end{array}$ & Buff-necked Ibis & - & A & $\begin{array}{l}\text { M C V } \\
\text { A H }\end{array}$ & jan-dec & - & - \\
\hline \multicolumn{8}{|l|}{ CICONIIDAE (2) } \\
\hline $\begin{array}{l}\text { Jabiru mycteria } \\
\text { (Lichtenstein, 1819) }\end{array}$ & Jabiru & AMG & $\mathrm{R}$ & M A H & jan-dec & - & - \\
\hline $\begin{array}{l}\text { Mycteria americana } \\
\text { Linnaeus, } 1758\end{array}$ & $\begin{array}{l}\text { American } \\
\text { Wood-Ibis }\end{array}$ & AMG & $\mathrm{E}$ & A H & jan-oct & - & - \\
\hline \multicolumn{8}{|l|}{ CATHARTIDAE (3) } \\
\hline $\begin{array}{l}\text { Cathartes aura } \\
\text { (Linnaeus, 1758) }\end{array}$ & Turkey Vulture & - & $\mathrm{C}$ & $\begin{array}{l}\text { M C V } \\
\text { A H }\end{array}$ & jan-dec & - & - \\
\hline $\begin{array}{l}\text { Cathartes burrovianus } \\
\text { Cassin, } 1845\end{array}$ & $\begin{array}{l}\text { Lesser Yellow- } \\
\text { headed Vulture }\end{array}$ & - & $\mathrm{R}$ & $\mathrm{C}$ & feb,may & - & - \\
\hline $\begin{array}{l}\text { Coragyps atratus } \\
\text { (Bechstein, 1793) }\end{array}$ & Black Vulture & - & A & $\begin{array}{l}\text { M C V } \\
\text { A H }\end{array}$ & jan-dec & - & - \\
\hline \multicolumn{8}{|l|}{ ACCIPITRIDAE (10) } \\
\hline $\begin{array}{l}\text { Leptodon cayanensis } \\
\text { (Latham, 1790) }\end{array}$ & Grey-headed Kite & - & $\mathrm{R}$ & M V & jan-dec & - & - \\
\hline $\begin{array}{l}\text { Elanus leucurus } \\
\text { (Vieillot, 1818) }\end{array}$ & White-tailed Kite & - & $\mathrm{O}$ & $\mathrm{C}$ & feb & - & - \\
\hline $\begin{array}{l}\text { Rostrhamus sociabilis } \\
\text { (Vieillot, 1817) }\end{array}$ & $\begin{array}{l}\text { Snail (Everglade) } \\
\text { Kite }\end{array}$ & - & $\mathrm{R}$ & A H & feb,may & - & - \\
\hline $\begin{array}{l}\text { Ictinia plumbea } \\
\text { (Gmelin, 1788) }\end{array}$ & Plumbeous Kite & - & $\mathrm{E}$ & M C V & aug-dec & - & - \\
\hline $\begin{array}{l}\text { Geranospiza caerulescens } \\
\text { (Vieillot, 1817) }\end{array}$ & Crane Hawk & - & $\mathrm{R}$ & $\mathrm{CH}$ & feb,dec & - & - \\
\hline $\begin{array}{l}\text { Buteogallus urubitinga } \\
\text { (Gmelin, 1788) }\end{array}$ & $\begin{array}{c}\text { Great Black } \\
\text { Hawk }\end{array}$ & - & $\mathrm{O}$ & M A & may & - & - \\
\hline $\begin{array}{l}\text { Heterospizias meridionalis } \\
\text { (Latham, 1790) }\end{array}$ & Savanna Hawk & - & $\mathrm{A}$ & C V A H & jan-dec & - & - \\
\hline $\begin{array}{l}\text { Rupornis magnirostris } \\
\text { (Gmelin, 1788) }\end{array}$ & Roadside Hawk & - & A & $\begin{array}{c}\text { M C V } \\
\text { A H }\end{array}$ & jan-dec & - & - \\
\hline $\begin{array}{l}\text { Buteo albicaudatus } \\
\text { Vieillot, } 1816\end{array}$ & $\begin{array}{l}\text { White-tailed } \\
\text { Hawk }\end{array}$ & - & $\mathrm{O}$ & C V & may & - & - \\
\hline
\end{tabular}


Table 1. Continued..

\begin{tabular}{|c|c|c|c|c|c|c|c|}
\hline Families and species & $\begin{array}{l}\text { Common } \\
\text { names }\end{array}$ & $\begin{array}{l}\text { Conservation } \\
\text { and endemism }\end{array}$ & Abundance & Habitat & $\begin{array}{l}\text { Month of } \\
\text { record }\end{array}$ & Reproduction & $\begin{array}{c}\text { Number } \\
\text { captured } \\
\text { birds } \\
\end{array}$ \\
\hline $\begin{array}{l}\text { Spizaetus melanoleucus } \\
\text { (Vieillot, 1816) }\end{array}$ & $\begin{array}{l}\text { Black-and-white } \\
\text { Hawk-Eagle }\end{array}$ & AMG & $\mathrm{R}$ & M C V & may,jul & - & - \\
\hline \multicolumn{8}{|l|}{ FALCONIDAE (6) } \\
\hline $\begin{array}{l}\text { Caracara plancus } \\
\text { (Miller, 1777) }\end{array}$ & Crested Caracara & - & A & $\begin{array}{c}\text { M C V } \\
\text { A H }\end{array}$ & jan-dec & - & - \\
\hline $\begin{array}{l}\text { Milvago chimachima } \\
\text { (Vieillot, 1816) }\end{array}$ & $\begin{array}{l}\text { Yellow-headed } \\
\text { Caracara }\end{array}$ & - & A & $\begin{array}{l}\text { M C V } \\
\text { A H }\end{array}$ & jan-dec & - & - \\
\hline $\begin{array}{l}\text { Herpetotheres cachinnans } \\
\text { (Linnaeus, 1758) }\end{array}$ & Laughing Falcon & - & A & $\begin{array}{c}\mathrm{M} \mathrm{C} \mathrm{V} \\
\mathrm{A} \mathrm{H}\end{array}$ & jan-dec & - & - \\
\hline $\begin{array}{l}\text { Micrastur semitorquatus } \\
\text { (Vieillot, 1817) }\end{array}$ & $\begin{array}{c}\text { Collared } \\
\text { Forest-Falcon }\end{array}$ & - & $\mathrm{R}$ & M C & $\begin{array}{l}\text { feb,apr, } \\
\text { jul }\end{array}$ & - & - \\
\hline $\begin{array}{l}\text { Falco sparverius } \\
\text { Linnaeus, } 1758\end{array}$ & American Kestrel & - & A & C A H & jan-dec & - & - \\
\hline $\begin{array}{l}\text { Falco femoralis } \\
\text { Temminck, } 1822\end{array}$ & $\begin{array}{l}\text { Aplomado } \\
\text { Falcon }\end{array}$ & - & $\mathrm{E}$ & C A H & jan-dec & - & - \\
\hline \multicolumn{8}{|l|}{ ARAMIDAE (1) } \\
\hline $\begin{array}{l}\text { Aramus guarauna } \\
\text { (Linnaeus, 1766) }\end{array}$ & Limpkin & - & $\mathrm{O}$ & A & apr & - & - \\
\hline \multicolumn{8}{|l|}{ RALLIDAE (2) } \\
\hline $\begin{array}{l}\text { Aramides ypecaha } \\
\text { (Vieillot, 1819) }\end{array}$ & Giant Wood-Rail & - & $\mathrm{R}$ & M A & jan-dec & - & - \\
\hline $\begin{array}{l}\text { Porzana albicollis } \\
\text { (Vieillot, 1819) }\end{array}$ & $\begin{array}{l}\text { Ash-throated } \\
\text { Crake }\end{array}$ & - & $\mathrm{E}$ & V A & jan-dec & - & - \\
\hline \multicolumn{8}{|l|}{ CARIAMIDAE (1) } \\
\hline $\begin{array}{l}\text { Cariama cristata } \\
\text { (Linnaeus, 1766) }\end{array}$ & $\begin{array}{l}\text { Red-legged } \\
\text { Seriema }\end{array}$ & - & A & C A H & jan-dec & jun & - \\
\hline \multicolumn{8}{|l|}{ CHARADRIIDAE (2) } \\
\hline $\begin{array}{l}\text { Vanellus cayanus } \\
\text { (Latham, 1790) }\end{array}$ & Cayenne Plover & - & $\mathrm{O}$ & A & aug & - & - \\
\hline \multicolumn{8}{|l|}{ LARIDAE (1) } \\
\hline $\begin{array}{l}\text { Vanellus chilensis } \\
\text { (Molina, 1782) }\end{array}$ & $\begin{array}{l}\text { Southern } \\
\text { Lapwing }\end{array}$ & - & A & C V A H & jan-dec & jan & 1 \\
\hline \multicolumn{8}{|l|}{ RECURVIROSTRIDAE (1) } \\
\hline $\begin{array}{l}\text { Himantopus melanurus } \\
\text { Vieillot, } 1817\end{array}$ & $\begin{array}{l}\text { White-backed } \\
\text { Stilt }\end{array}$ & - & $\mathrm{R}$ & A & jan-dec & - & - \\
\hline \multicolumn{8}{|l|}{ SCOLOPACIDAE (2) } \\
\hline $\begin{array}{l}\text { Tringa solitaria } \\
\text { Wilson, } 1813\end{array}$ & $\begin{array}{l}\text { Solitary } \\
\text { Sandpiper }\end{array}$ & - & $\mathrm{O}$ & A H & dec & - & - \\
\hline $\begin{array}{l}\text { Tringa melanoleuca } \\
\text { (Gmelin, 1789) }\end{array}$ & $\begin{array}{l}\text { Greater } \\
\text { Yellowlegs }\end{array}$ & - & $\mathrm{O}$ & A & jan & - & - \\
\hline \multicolumn{8}{|l|}{ JACANIDAE (1) } \\
\hline $\begin{array}{l}\text { Jacana jacana } \\
\text { (Linnaeus, 1766) }\end{array}$ & Wattled Jacana & - & $\mathrm{C}$ & A & feb-aug & - & - \\
\hline $\begin{array}{l}\text { Phaetusa simplex } \\
\text { (Gmelin, 1789) }\end{array}$ & Large-billed Tern & - & $\mathrm{O}$ & A & aug & - & - \\
\hline \multicolumn{8}{|l|}{ COLUMBIDAE (9) } \\
\hline $\begin{array}{l}\text { Columbina talpacoti } \\
\text { (Temminck, 1811) }\end{array}$ & $\begin{array}{c}\text { Ruddy } \\
\text { Ground-Dove }\end{array}$ & - & A & $\mathrm{CV}$ & jan-dec & - & 2 \\
\hline $\begin{array}{l}\text { Columbina squammata } \\
\text { (Lesson, 1831) }\end{array}$ & Scaled Dove & - & A & $\mathrm{CV}$ & jan-dec & - & 3 \\
\hline $\begin{array}{l}\text { Columbina picui } \\
\text { (Temminck, 1813) }\end{array}$ & $\begin{array}{c}\text { Picui } \\
\text { Ground-Dove }\end{array}$ & - & $\mathrm{E}$ & $\mathrm{CH}$ & oct-mar & - & - \\
\hline
\end{tabular}


Table 1. Continued...

\begin{tabular}{|c|c|c|c|c|c|c|c|}
\hline Families and species & $\begin{array}{l}\text { Common } \\
\text { names }\end{array}$ & $\begin{array}{c}\text { Conservation } \\
\text { and endemism }\end{array}$ & Abundance & Habitat & $\begin{array}{l}\text { Month of } \\
\text { record }\end{array}$ & Reproduction & $\begin{array}{c}\text { Number } \\
\text { captured } \\
\text { birds }\end{array}$ \\
\hline $\begin{array}{l}\text { Claravis pretiosa } \\
\text { (Ferrari-Perez, 1886) }\end{array}$ & $\begin{array}{c}\text { Blue } \\
\text { Ground-Dove }\end{array}$ & - & $\mathrm{R}$ & M & dec-apr & - & 3 \\
\hline $\begin{array}{l}\text { Uropelia campestris } \\
(\text { Spix, 1825) }\end{array}$ & $\begin{array}{l}\text { Long-tailed } \\
\text { Ground-Dove }\end{array}$ & - & $\mathrm{R}$ & $\mathrm{CH}$ & jan-dec & - & - \\
\hline $\begin{array}{l}\text { Patagioenas picazuro } \\
\text { (Temminck, 1813) }\end{array}$ & Picazuro Pigeon & - & A & M C V & jan-dec & - & - \\
\hline $\begin{array}{l}\text { Patagioenas cayennensis } \\
\text { (Bonnaterre, 1792) }\end{array}$ & $\begin{array}{l}\text { Pale-vented } \\
\text { Pigeon }\end{array}$ & - & $\mathrm{E}$ & M C V & jan-dec & - & - \\
\hline $\begin{array}{l}\text { Zenaida auriculata } \\
\text { (Des Murs, 1847) }\end{array}$ & Eared Dove & - & $\mathrm{R}$ & $\mathrm{C}$ & oct, dec & - & - \\
\hline $\begin{array}{l}\text { Leptotila verreauxi } \\
\text { Bonaparte, } 1855\end{array}$ & $\begin{array}{l}\text { White-tipped } \\
\text { Dove }\end{array}$ & - & A & M C V & jan-dec & - & 6 \\
\hline \multicolumn{8}{|l|}{ PSITTACIDAE (11) } \\
\hline $\begin{array}{l}\text { Ara ararauna } \\
\text { (Linnaeus, 1758) }\end{array}$ & $\begin{array}{c}\text { Blue-and-yellow } \\
\text { Macaw }\end{array}$ & AMG & A & M C V & jan-dec & jun-aug & - \\
\hline $\begin{array}{l}\text { Orthopsittaca manilata } \\
\text { (Boddaert, 1783) }\end{array}$ & $\begin{array}{c}\text { Red-bellied } \\
\text { Macaw }\end{array}$ & - & $\mathrm{E}$ & M C V & jan-dec & jun & - \\
\hline $\begin{array}{l}\text { Diopsittaca nobilis } \\
\text { (Linnaeus, 1758) }\end{array}$ & $\begin{array}{l}\text { Red-shouldered } \\
\text { Macaw }\end{array}$ & - & $\mathrm{C}$ & M C V & jan-dec & - & - \\
\hline $\begin{array}{l}\text { Aratinga leucophthalma } \\
\text { (Statius Muller, 1776) }\end{array}$ & $\begin{array}{l}\text { White-eyed } \\
\text { Parakeet }\end{array}$ & - & $\mathrm{E}$ & M C & jan-dec & - & - \\
\hline $\begin{array}{l}\text { Aratinga aurea } \\
\text { (Gmelin, 1788) }\end{array}$ & $\begin{array}{l}\text { Peach-fronted } \\
\text { Parakeet }\end{array}$ & - & A & M C V & jan-dec & - & 1 \\
\hline $\begin{array}{l}\text { Aratinga cactorum } \\
\text { (Kuhl, 1820) }\end{array}$ & $\begin{array}{c}\text { Caatinga } \\
\text { (Cactus) Parakeet }\end{array}$ & $\mathrm{ECa}$ & A & M C V & jan-dec & - & - \\
\hline $\begin{array}{l}\text { Forpus xanthopterygius } \\
\text { (Spix, 1824) }\end{array}$ & $\begin{array}{c}\text { Blue-winged } \\
\text { Parrotlet }\end{array}$ & - & A & M C V & jan-dec & - & - \\
\hline $\begin{array}{l}\text { Brotogeris chiriri } \\
\text { (Vieillot, 1818) }\end{array}$ & $\begin{array}{l}\text { Yellow-chev- } \\
\text { roned Parakeet }\end{array}$ & - & A & M C V & jan-dec & - & 1 \\
\hline $\begin{array}{l}\text { Alipiopsitta xanthops } \\
\text { (Spix, 1824) }\end{array}$ & $\begin{array}{l}\text { Yellow-faced } \\
\text { Parrot }\end{array}$ & AMG & A & $\mathrm{CV}$ & jan-dec & - & - \\
\hline $\begin{array}{l}\text { Pionus maximiliani } \\
\text { (Kuhl, 1820) }\end{array}$ & $\begin{array}{l}\text { Scaly-headed } \\
\text { Parrot }\end{array}$ & - & $\mathrm{C}$ & $\mathrm{CV}$ & jan-dec & - & - \\
\hline $\begin{array}{l}\text { Amazona aestiva } \\
\text { (Linnaeus, 1758) }\end{array}$ & $\begin{array}{l}\text { Turquoise-front- } \\
\text { ed Parrot }\end{array}$ & - & A & $\mathrm{CV}$ & jan-dec & - & - \\
\hline \multicolumn{8}{|l|}{ CUCULIDAE (5) } \\
\hline $\begin{array}{l}\text { Piaya cayana } \\
\text { (Linnaeus, 1766) }\end{array}$ & Squirrel Cuckoo & - & $\mathrm{A}$ & M C V & jan-dec & - & - \\
\hline $\begin{array}{l}\text { Coccyzus melacoryphus } \\
\text { Vieillot, } 1817\end{array}$ & $\begin{array}{l}\text { Dark-billed } \\
\text { Cuckoo }\end{array}$ & - & $\mathrm{O}$ & $\mathrm{C}$ & mar & - & - \\
\hline $\begin{array}{l}\text { Crotophaga ani } \\
\text { Linnaeus, } 1758\end{array}$ & $\begin{array}{l}\text { Smooth-billed } \\
\text { Ani }\end{array}$ & - & A & $\mathrm{CAH}$ & jan-dec & - & 1 \\
\hline $\begin{array}{l}\text { Guira guira } \\
\text { (Gmelin, 1788) }\end{array}$ & Guira Cuckoo & - & A & $\mathrm{CV}$ & jan-dec & - & - \\
\hline $\begin{array}{l}\text { Tapera naevia } \\
\text { (Linnaeus, 1766) }\end{array}$ & Striped Cuckoo & - & $\mathrm{C}$ & M C & jan-dec & - & - \\
\hline \multicolumn{8}{|l|}{ TYTONIDAE (1) } \\
\hline $\begin{array}{l}\text { Tyto alba } \\
\text { (Scopoli, 1769) }\end{array}$ & Barn Owl & - & $\mathrm{O}$ & M H & may & may & - \\
\hline STRIGIDAE (5) & & & & & & - & - \\
\hline $\begin{array}{l}\text { Megascops choliba } \\
\text { (Vieillot, 1817) }\end{array}$ & $\begin{array}{c}\text { Tropical } \\
\text { Screech-Owl }\end{array}$ & - & $\mathrm{C}$ & M C V & jan-dec & - & - \\
\hline
\end{tabular}


Table 1. Continued..

\begin{tabular}{|c|c|c|c|c|c|c|c|}
\hline Families and species & $\begin{array}{l}\text { Common } \\
\text { names }\end{array}$ & $\begin{array}{c}\text { Conservation } \\
\text { and endemism }\end{array}$ & Abundance & Habitat & $\begin{array}{l}\text { Month of } \\
\text { record }\end{array}$ & Reproduction & $\begin{array}{c}\text { Number } \\
\text { captured } \\
\text { birds }\end{array}$ \\
\hline $\begin{array}{l}\text { Bubo virginianus } \\
\text { (Gmelin, 1788) }\end{array}$ & $\begin{array}{l}\text { Great Horned } \\
\text { Owl }\end{array}$ & - & $\mathrm{R}$ & $\mathrm{CV}$ & jan-dec & - & - \\
\hline $\begin{array}{l}\text { Glaucidium brasilianum } \\
\text { (Gmelin, 1788) }\end{array}$ & $\begin{array}{l}\text { Ferruginous } \\
\text { Pygmy-Owl }\end{array}$ & - & A & M C V & jan-dec & - & 5 \\
\hline $\begin{array}{l}\text { Athene cunicularia } \\
\text { (Molina, 1782) }\end{array}$ & Burrowing Owl & - & $\mathrm{C}$ & $\mathrm{CH}$ & jan-dec & - & - \\
\hline $\begin{array}{l}\text { Rhinoptynx clamator } \\
\text { (Vieillot, 1808) }\end{array}$ & Striped Owl & - & $\mathrm{O}$ & $\mathrm{H}$ & jul & jun & - \\
\hline \multicolumn{8}{|l|}{ NYCTIBIIDAE (1) } \\
\hline $\begin{array}{l}\text { Nyctibius griseus } \\
\text { (Gmelin, 1789) }\end{array}$ & Common Potoo & - & $\mathrm{E}$ & M C & jan-dec & - & - \\
\hline \multicolumn{8}{|l|}{ CAPRIMULGIDAE (6) } \\
\hline $\begin{array}{l}\text { Chordeiles pusillus } \\
\text { Gould, } 1861\end{array}$ & Least Nighthawk & - & $\mathrm{C}$ & $\mathrm{CV}$ & jan-dec & - & - \\
\hline $\begin{array}{l}\text { Podager nacunda } \\
\text { (Vieillot, 1817) }\end{array}$ & $\begin{array}{l}\text { Nacunda } \\
\text { Nighthawk }\end{array}$ & - & $\mathrm{O}$ & $\mathrm{CA}$ & dec & - & - \\
\hline $\begin{array}{l}\text { Nyctidromus albicollis } \\
\text { (Gmelin, 1789) }\end{array}$ & Pauraque & - & A & C V A H & jan-dec & - & - \\
\hline $\begin{array}{l}\text { Caprimulgus rufus } \\
\text { Boddaert, } 1783\end{array}$ & Rufous Nightjar & - & $\mathrm{O}$ & $\mathrm{H}$ & feb & feb & 1 \\
\hline $\begin{array}{l}\text { Caprimulgus parvulus } \\
\text { Gould, } 1837\end{array}$ & Little Nightjar & - & $\mathrm{R}$ & $\mathrm{CH}$ & aug-oct & - & - \\
\hline $\begin{array}{l}\text { Hydropsalis torquata } \\
\text { (Gmelin, 1789) }\end{array}$ & $\begin{array}{l}\text { Scissor-tailed } \\
\text { Nightjar }\end{array}$ & - & $\mathrm{E}$ & $\mathrm{CV}$ & jan-dec & - & - \\
\hline \multicolumn{8}{|l|}{ APODIDAE (3) } \\
\hline $\begin{array}{l}\text { Streptoprocne zonaris } \\
\text { (Shaw, 1796) }\end{array}$ & $\begin{array}{l}\text { White-collared } \\
\text { Swift }\end{array}$ & - & $\mathrm{O}$ & $\mathrm{CA}$ & feb & - & - \\
\hline $\begin{array}{l}\text { Chaetura meridionalis } \\
\text { Hellmayr, } 1907\end{array}$ & $\begin{array}{l}\text { Ashy-tailed } \\
\text { (Sick's) Swift }\end{array}$ & - & $\mathrm{R}$ & $\mathrm{C}$ & $\begin{array}{l}\text { jul,oct, } \\
\text { dec }\end{array}$ & - & - \\
\hline $\begin{array}{l}\text { Tachornis squamata } \\
\text { (Cassin, 1853) }\end{array}$ & $\begin{array}{l}\text { Fork-tailed } \\
\text { Palm-Swift }\end{array}$ & - & $\mathrm{C}$ & $\mathrm{CV}$ A H & jul-apr & - & - \\
\hline \multicolumn{8}{|l|}{ TROCHILIDAE (13) } \\
\hline $\begin{array}{l}\text { Phaethornis pretrei } \\
\text { (Lesson \& Delattre, 1839) }\end{array}$ & Planalto Hermit & - & $\mathrm{C}$ & M C V & oct-jun & oct & 13 \\
\hline $\begin{array}{l}\text { Eupetomena macroura } \\
\text { (Gmelin, 1788) }\end{array}$ & $\begin{array}{l}\text { Swallow-tailed } \\
\text { Hummingbird }\end{array}$ & - & A & M C V & jan-dec & - & 21 \\
\hline $\begin{array}{l}\text { Florisuga fusca } \\
\text { (Vieillot, 1817) }\end{array}$ & Black Jacobin & Ema & $\mathrm{O}$ & M C & aug & - & - \\
\hline $\begin{array}{l}\text { Colibri serrirostris } \\
\text { (Vieillot, 1816) }\end{array}$ & $\begin{array}{l}\text { White-vented } \\
\text { Violetear }\end{array}$ & - & $\mathrm{E}$ & M C V & may-oct & - & - \\
\hline $\begin{array}{l}\text { Anthracothorax nigricollis } \\
\text { (Vieillot, 1817) }\end{array}$ & $\begin{array}{l}\text { Black-throated } \\
\text { Mango }\end{array}$ & - & $\mathrm{R}$ & M C & $\begin{array}{l}\text { may,aug, } \\
\text { dec }\end{array}$ & - & - \\
\hline $\begin{array}{l}\text { Chrysolampis mosquitus } \\
\text { (Linnaeus, 1758) }\end{array}$ & $\begin{array}{l}\text { Ruby-topaz } \\
\text { Hummingbird }\end{array}$ & - & $\mathrm{O}$ & $\mathrm{C}$ & aug & - & - \\
\hline $\begin{array}{l}\text { Chlorostilbon lucidus } \\
\text { (Shaw, 1812) }\end{array}$ & $\begin{array}{l}\text { Glittering-bellied } \\
\text { Emerald }\end{array}$ & - & $\mathrm{C}$ & M C V & apr-oct & - & 11 \\
\hline $\begin{array}{l}\text { Thalurania furcata } \\
\text { (Gmelin, 1788) }\end{array}$ & $\begin{array}{l}\text { Fork-tailed } \\
\text { Woodnymph }\end{array}$ & - & $\mathrm{C}$ & M & oct-may & - & 15 \\
\hline $\begin{array}{l}\text { Amazilia versicolor } \\
\text { (Vieillot, 1818) }\end{array}$ & $\begin{array}{l}\text { Versicoloured } \\
\text { Emerald }\end{array}$ & - & $\mathrm{R}$ & M C & aug,oct & - & 4 \\
\hline $\begin{array}{l}\text { Amazilia fimbriata } \\
\text { (Gmelin, 1788) }\end{array}$ & $\begin{array}{l}\text { Glittering-throat- } \\
\text { ed Emerald }\end{array}$ & - & $\mathrm{C}$ & $\begin{array}{c}\text { M C V } \\
\text { A H }\end{array}$ & jan-dec & - & 77 \\
\hline
\end{tabular}


Table 1. Continued...

\begin{tabular}{|c|c|c|c|c|c|c|c|}
\hline Families and species & $\begin{array}{l}\text { Common } \\
\text { names }\end{array}$ & $\begin{array}{c}\text { Conservation } \\
\text { and endemism }\end{array}$ & Abundance & Habitat & $\begin{array}{l}\text { Month of } \\
\text { record }\end{array}$ & Reproduction & $\begin{array}{c}\text { Number } \\
\text { captured } \\
\text { birds }\end{array}$ \\
\hline $\begin{array}{l}\text { Heliactin bilophus } \\
\text { (Temminck, 1820) }\end{array}$ & Horned Sungem & - & $\mathrm{O}$ & $\mathrm{C}$ & jul & - & - \\
\hline $\begin{array}{l}\text { Heliomaster squamosus } \\
\text { (Temminck, 1823) }\end{array}$ & $\begin{array}{l}\text { Stripe-breasted } \\
\text { Starthroat }\end{array}$ & - & $\mathrm{O}$ & $\mathrm{C}$ & jul & - & - \\
\hline $\begin{array}{l}\text { Calliphlox amethystina } \\
\text { (Boddaert, 1783) }\end{array}$ & $\begin{array}{l}\text { Amethyst } \\
\text { Woodstar }\end{array}$ & - & $\mathrm{O}$ & $\mathrm{C}$ & mar & - & - \\
\hline \multicolumn{8}{|l|}{ TROGONIDAE (1) } \\
\hline $\begin{array}{l}\text { Trogon surrucura } \\
\text { Vieillot, } 1817\end{array}$ & Surucua Trogon & - & A & M & jan-dec & - & - \\
\hline \multicolumn{8}{|l|}{ ALCEDINIDAE (3) } \\
\hline $\begin{array}{l}\text { Megaceryle torquata } \\
\text { (Linnaeus, 1766) }\end{array}$ & $\begin{array}{l}\text { Ringed } \\
\text { Kingfisher }\end{array}$ & - & $\mathrm{C}$ & V A & jan-dec & - & - \\
\hline $\begin{array}{l}\text { Chloroceryle amazona (Latham, } \\
\text { 1790) }\end{array}$ & $\begin{array}{l}\text { Amazon } \\
\text { Kingfisher }\end{array}$ & - & $\mathrm{E}$ & V A & jan-aug & - & - \\
\hline $\begin{array}{l}\text { Chloroceryle americana } \\
\text { (Gmelin, 1788) }\end{array}$ & Green Kingfisher & - & $\mathrm{E}$ & V A & apr-sep & - & - \\
\hline \multicolumn{8}{|l|}{ GALBULIDAE (1) } \\
\hline $\begin{array}{l}\text { Galbula ruficauda } \\
\text { Cuvier, } 1816\end{array}$ & $\begin{array}{l}\text { Rufous-tailed } \\
\text { Jacamar }\end{array}$ & - & A & M C V A & jan-dec & - & 4 \\
\hline \multicolumn{8}{|l|}{ BUCCONIDAE (3) } \\
\hline $\begin{array}{l}\text { Nystalus chacuru } \\
\text { (Vieillot, 1816) }\end{array}$ & $\begin{array}{l}\text { White-eared } \\
\text { Puffbird }\end{array}$ & - & $\mathrm{C}$ & $\mathrm{CV} \mathrm{A} \mathrm{H}$ & jan-dec & - & - \\
\hline $\begin{array}{l}\text { Nystalus maculatus } \\
\text { (Gmelin, 1788) }\end{array}$ & $\begin{array}{l}\text { Spot-backed } \\
\text { Puffbird }\end{array}$ & - & $\mathrm{R}$ & M C & aug-apr & - & 7 \\
\hline $\begin{array}{l}\text { Nonnula rubecula } \\
\text { (Spix, 1824) }\end{array}$ & $\begin{array}{l}\text { Rusty-breasted } \\
\text { Nunlet }\end{array}$ & - & $\mathrm{E}$ & M & aug-mar & oct & 10 \\
\hline \multicolumn{8}{|l|}{ RAMPHASTIDAE (1) } \\
\hline $\begin{array}{l}\text { Ramphastos toco Statius } \\
\text { Muller, } 1776\end{array}$ & Toco Toucan & - & A & $\begin{array}{c}\text { M C V } \\
\text { A H }\end{array}$ & jan-dec & oct & - \\
\hline \multicolumn{8}{|l|}{ PICIDAE (10) } \\
\hline $\begin{array}{l}\text { Picumnus albosquamatus } \\
\text { d'Orbigny, } 1840\end{array}$ & $\begin{array}{c}\text { White-wedged } \\
\text { (Arrowhead) } \\
\text { Piculet }\end{array}$ & - & $\mathrm{E}$ & M C & jan-dec & oct & 3 \\
\hline $\begin{array}{l}\text { Melanerpes candidus } \\
\text { (Otto, 1796) }\end{array}$ & $\begin{array}{c}\text { White } \\
\text { Woodpecker }\end{array}$ & - & $\mathrm{E}$ & $\begin{array}{c}\text { M C V } \\
\text { A H }\end{array}$ & dec-aug & - & - \\
\hline $\begin{array}{l}\text { Veniliornis passerinus } \\
\text { (Linnaeus, 1766) }\end{array}$ & $\begin{array}{c}\text { Little } \\
\text { Woodpecker }\end{array}$ & - & $\mathrm{C}$ & M C & jan-dec & jul & 2 \\
\hline $\begin{array}{l}\text { Veniliornis mixtus } \\
\text { (Boddaert, 1783) }\end{array}$ & $\begin{array}{l}\text { Checkered } \\
\text { Woodpecker }\end{array}$ & - & $\mathrm{R}$ & $\mathrm{C}$ & may,jul & - & - \\
\hline $\begin{array}{l}\text { Piculus chrysochloros } \\
\text { (Vieillot, 1818) }\end{array}$ & $\begin{array}{l}\text { Golden-green } \\
\text { Woodpecker }\end{array}$ & - & $\mathrm{E}$ & M C & dec-aug & - & - \\
\hline $\begin{array}{l}\text { Colaptes melanochloros } \\
\text { (Gmelin, 1788) }\end{array}$ & $\begin{array}{l}\text { Green-barred } \\
\text { Flicker } \\
\text { (Woodpecker) }\end{array}$ & - & $\mathrm{C}$ & $\begin{array}{c}\text { M C V } \\
\text { A H }\end{array}$ & jan-dec & - & - \\
\hline $\begin{array}{l}\text { Colaptes campestris } \\
\text { (Vieillot, 1818) }\end{array}$ & Campo Flicker & - & A & C V A H & jan-dec & oct & - \\
\hline $\begin{array}{l}\text { Celeus flavescens } \\
\text { (Gmelin, 1788) }\end{array}$ & $\begin{array}{l}\text { Blond-crested } \\
\text { Woodpecker }\end{array}$ & - & $\mathrm{E}$ & M C & jan-dec & dec & 1 \\
\hline $\begin{array}{l}\text { Dryocopus lineatus } \\
\text { (Linnaeus, 1766) }\end{array}$ & $\begin{array}{c}\text { Lineated } \\
\text { Woodpecker }\end{array}$ & - & A & $\begin{array}{c}\text { M C V } \\
\text { A H }\end{array}$ & jan-dec & - & 2 \\
\hline $\begin{array}{l}\text { Campephilus melanoleucos } \\
\text { (Gmelin, 1788) }\end{array}$ & $\begin{array}{l}\text { Crimson-crested } \\
\text { Woodpecker }\end{array}$ & - & $\mathrm{E}$ & M C & jan-dec & - & - \\
\hline
\end{tabular}


Table 1. Continued..

\begin{tabular}{|c|c|c|c|c|c|c|c|}
\hline Families and species & $\begin{array}{l}\text { Common } \\
\text { names }\end{array}$ & $\begin{array}{l}\text { Conservation } \\
\text { and endemism }\end{array}$ & Abundance & Habitat & $\begin{array}{l}\text { Month of } \\
\text { record }\end{array}$ & Reproduction & $\begin{array}{c}\text { Number } \\
\text { captured } \\
\text { birds }\end{array}$ \\
\hline \multicolumn{8}{|l|}{ THAMNOPHILIDAE (6) } \\
\hline $\begin{array}{l}\text { Taraba major } \\
\text { (Vieillot, 1816) }\end{array}$ & Great Antshrike & - & A & $\begin{array}{c}\text { M C V } \\
\text { A H }\end{array}$ & jan-dec & - & 2 \\
\hline $\begin{array}{l}\text { Thamnophilus doliatus } \\
\text { (Linnaeus, 1764) }\end{array}$ & Barred Antshrike & - & $\mathrm{R}$ & $\mathrm{C}$ & jan-dec & - & - \\
\hline $\begin{array}{l}\text { Thamnophilus pelzelni } \\
\text { Hellmayr, } 1924\end{array}$ & $\begin{array}{l}\text { Planalto } \\
\text { Slaty-Antshrike }\end{array}$ & - & A & M C & jan-dec & aug-may & 32 \\
\hline $\begin{array}{l}\text { Herpsilochmus atricapillus } \\
\text { Pelzeln, } 1868\end{array}$ & $\begin{array}{l}\text { Black-capped } \\
\text { Antwren }\end{array}$ & - & A & M & jan-dec & - & 2 \\
\hline $\begin{array}{l}\text { Herpsilochmus longirostris } \\
\text { Pelzeln, } 1868\end{array}$ & $\begin{array}{l}\text { Large-billed } \\
\text { Antwren }\end{array}$ & $\mathrm{ECe}$ & $\mathrm{E}$ & $\mathrm{V}$ & jan-dec & - & - \\
\hline $\begin{array}{l}\text { Formicivora rufa } \\
\text { (Wied, 1831) }\end{array}$ & $\begin{array}{l}\text { Rusty-backed } \\
\text { Antwren }\end{array}$ & - & $\mathrm{R}$ & M V & jan-dec & - & - \\
\hline \multicolumn{8}{|l|}{ DENDROCOLAPTIDAE (6) } \\
\hline $\begin{array}{l}\text { Sittasomus griseicapillus } \\
\text { (Vieillot, 1818) }\end{array}$ & $\begin{array}{l}\text { Olivaceous } \\
\text { Woodcreeper }\end{array}$ & - & A & M V & jan-dec & sep-feb & 13 \\
\hline $\begin{array}{l}\text { Xiphocolaptes albicollis } \\
\text { (Vieillot, 1818) }\end{array}$ & $\begin{array}{l}\text { White-throated } \\
\text { Woodcreeper }\end{array}$ & - & $\mathrm{O}$ & M & jun & - & - \\
\hline $\begin{array}{l}\text { Dendrocolaptes platyrostris } \\
\text { Spix, } 1825\end{array}$ & $\begin{array}{c}\text { Planalto } \\
\text { Woodcreeper }\end{array}$ & - & $\mathrm{C}$ & M V & jan-dec & feb & 14 \\
\hline $\begin{array}{l}\text { Lepidocolaptes angustirostris } \\
\text { (Vieillot, 1818) }\end{array}$ & $\begin{array}{l}\text { Narrow-billed } \\
\text { Woodcreeper }\end{array}$ & - & A & M C V & jan-dec & mar/oct-dec & - \\
\hline $\begin{array}{l}\text { Lepidocolaptes wagleri } \\
\text { (Spix, 1824) }\end{array}$ & $\begin{array}{c}\text { Wagler's } \\
\text { Woodcreeper }\end{array}$ & - & $\mathrm{E}$ & M & jan-dec & oct & 5 \\
\hline $\begin{array}{l}\text { Campylorhamphus trochilirostris } \\
\text { (Lichtenstein, 1820) }\end{array}$ & $\begin{array}{l}\text { Red-billed } \\
\text { Scythebill }\end{array}$ & - & $\mathrm{E}$ & M & jan-dec & - & - \\
\hline \multicolumn{8}{|l|}{ FURNARIIDAE (12) } \\
\hline $\begin{array}{l}\text { Furnarius figulus } \\
\text { (Lichtenstein, 1823) }\end{array}$ & $\begin{array}{c}\text { Wing-banded } \\
\text { (Band-tailed) } \\
\text { Hornero }\end{array}$ & - & $\mathrm{E}$ & A & jan-dec & - & - \\
\hline $\begin{array}{l}\text { Furnarius leucopus } \\
\text { Swainson, } 1838\end{array}$ & $\begin{array}{l}\text { Pale-legged } \\
\text { Hornero }\end{array}$ & - & $\mathrm{E}$ & C V A & jan-dec & oct & 1 \\
\hline $\begin{array}{l}\text { Furnarius rufus } \\
\text { (Gmelin, 1788) }\end{array}$ & Rufous Hornero & - & A & C V A H & jan-dec & dec & 1 \\
\hline $\begin{array}{l}\text { Schoeniophylax phryganophilus } \\
\text { (Vieillot, 1817) }\end{array}$ & Chotoy Spinetail & - & $\mathrm{O}$ & $\mathrm{C}$ & jul & - & - \\
\hline $\begin{array}{l}\text { Synallaxis frontalis } \\
\text { Pelzeln, } 1859\end{array}$ & $\begin{array}{l}\text { Sooty-fronted } \\
\text { Spinetail }\end{array}$ & - & A & M C V & jan-dec & feb-apr / nov & - \\
\hline $\begin{array}{l}\text { Synallaxis albescens } \\
\text { Temminck, } 1823\end{array}$ & $\begin{array}{l}\text { Pale-breasted } \\
\text { Spinetail }\end{array}$ & - & $\mathrm{R}$ & $\mathrm{CH}$ & jan-dec & - & - \\
\hline $\begin{array}{l}\text { Synallaxis scutata } \\
\text { Sclater, } 1859\end{array}$ & $\begin{array}{l}\text { Ochre-cheeked } \\
\text { Spinetail }\end{array}$ & - & A & M A & jan-dec & dec-feb & 12 \\
\hline $\begin{array}{l}\text { Cranioleuca vulpina } \\
\text { (Pelzeln, 1856) }\end{array}$ & $\begin{array}{l}\text { Rusty-backed } \\
\text { Spinetail }\end{array}$ & - & $\mathrm{O}$ & M C & feb & - & - \\
\hline $\begin{array}{l}\text { Phacellodomus rufifrons } \\
\text { (Wied, 1821) }\end{array}$ & $\begin{array}{l}\text { Rufous-fronted } \\
\text { Thornbird }\end{array}$ & - & A & $\mathrm{CH}$ & jan-dec & - & - \\
\hline $\begin{array}{l}\text { Phacellodomus ruber } \\
\text { (Vieillot, 1817) }\end{array}$ & $\begin{array}{l}\text { Greater (Yellow- } \\
\text { eyed) Thornbird }\end{array}$ & - & $\mathrm{E}$ & $\mathrm{CV}$ & jan-dec & oct & 2 \\
\hline $\begin{array}{l}\text { Hylocryptus rectirostris } \\
\text { (Wied, 1831) }\end{array}$ & $\begin{array}{l}\text { Chestnut } \\
\text { (Henna)-capped } \\
\text { Foliage-gleaner }\end{array}$ & $\mathrm{ECe}$ & A & M & jan-dec & dec & 3 \\
\hline
\end{tabular}


Table 1. Continued...

\begin{tabular}{|c|c|c|c|c|c|c|c|}
\hline Families and species & $\begin{array}{l}\text { Common } \\
\text { names }\end{array}$ & $\begin{array}{c}\text { Conservation } \\
\text { and endemism }\end{array}$ & Abundance & Habitat & $\begin{array}{l}\text { Month of } \\
\text { record }\end{array}$ & Reproduction & $\begin{array}{c}\text { Number } \\
\text { captured } \\
\text { birds }\end{array}$ \\
\hline $\begin{array}{l}\text { Xenops rutilans } \\
\text { Temminck, } 1821\end{array}$ & Streaked Xenops & - & $\mathrm{E}$ & M C & jan-dec & - & - \\
\hline \multicolumn{8}{|l|}{ TYRANNIDAE (49) } \\
\hline $\begin{array}{l}\text { Leptopogon amaurocephalus } \\
\text { Tschudi, } 1846\end{array}$ & $\begin{array}{l}\text { Sepia-capped } \\
\text { Flycatcher }\end{array}$ & - & $\mathrm{C}$ & M C & oct-jun & - & 11 \\
\hline $\begin{array}{l}\text { Hemitriccus striaticollis } \\
\text { (Lafresnaye, 1853) }\end{array}$ & $\begin{array}{l}\text { Stripe-necked } \\
\text { Tody-Tyrant }\end{array}$ & - & $\mathrm{R}$ & $\mathrm{V}$ & jan-dec & - & 2 \\
\hline $\begin{array}{l}\text { Hemitriccus margaritaceiventer } \\
\text { (d'Orbigny \& Lafresnaye, 1837) }\end{array}$ & $\begin{array}{l}\text { Pearly-vented } \\
\text { Tody-tyrant }\end{array}$ & - & $\mathrm{E}$ & M C V & aug-apr & sep-oct & 6 \\
\hline $\begin{array}{l}\text { Poecilotriccus latirostris } \\
\text { (Pelzeln, 1868) }\end{array}$ & $\begin{array}{l}\text { Rusty-fronted } \\
\text { Tody-Flycatcher }\end{array}$ & - & $\mathrm{R}$ & M & feb,may & - & 1 \\
\hline $\begin{array}{l}\text { Todirostrum cinereum } \\
\text { (Linnaeus, 1766) }\end{array}$ & $\begin{array}{c}\text { Common } \\
\text { Tody-Flycatcher }\end{array}$ & - & $\mathrm{E}$ & M C & jan-dec & - & - \\
\hline $\begin{array}{l}\text { Phyllomyias fasciatus } \\
\text { (Thunberg, 1822) }\end{array}$ & $\begin{array}{c}\text { Planalto } \\
\text { Tyrannulet }\end{array}$ & - & $\mathrm{C}$ & M C & jan-dec & - & - \\
\hline $\begin{array}{l}\text { Myiopagis caniceps } \\
\text { (Swainson, 1835) }\end{array}$ & Grey Elaenia & - & $\mathrm{E}$ & M & jan-dec & - & - \\
\hline $\begin{array}{l}\text { Myiopagis viridicata } \\
\text { (Vieillot, 1817) }\end{array}$ & Greenish Elaenia & - & $\mathrm{E}$ & M C & oct-may & oct-jan & 20 \\
\hline $\begin{array}{l}\text { Elaenia flavogaster } \\
\text { (Thunberg, 1822) }\end{array}$ & $\begin{array}{l}\text { Yellow-bellied } \\
\text { Elaenia }\end{array}$ & - & $\mathrm{C}$ & C V A H & jan-dec & - & 2 \\
\hline $\begin{array}{l}\text { Elaenia spectabilis } \\
\text { Pelzeln, } 1868\end{array}$ & Large Elaenia & - & $\mathrm{R}$ & M C & oct,dec & - & - \\
\hline $\begin{array}{l}\text { Elaenia parvirostris } \\
\text { Pelzeln, } 1868\end{array}$ & $\begin{array}{l}\text { Small-billed } \\
\text { Elaenia }\end{array}$ & - & $\mathrm{O}$ & M & may & - & 1 \\
\hline $\begin{array}{l}\text { Elaenia cristata } \\
\text { Pelzeln, } 1868\end{array}$ & $\begin{array}{l}\text { Plain-crested } \\
\text { Elaenia }\end{array}$ & - & $\mathrm{R}$ & $\mathrm{C}$ & mar-oct & - & - \\
\hline $\begin{array}{l}\text { Elaenia chiriquensis } \\
\text { Lawrence, } 1865\end{array}$ & Lesser Elaenia & - & $\mathrm{E}$ & $\mathrm{C}$ & aug-apr & dec & 2 \\
\hline $\begin{array}{l}\text { Camptostoma obsoletum } \\
\text { (Temminck, 1824) }\end{array}$ & $\begin{array}{l}\text { Southern Beard- } \\
\text { less Tyrannulet }\end{array}$ & - & A & M C & jan-dec & - & 9 \\
\hline Suiriri suiriri (Vieillot, 1818) & Chaco Suiriri & $\mathrm{ECe}$ & $\mathrm{E}$ & $\mathrm{C}$ & jan-dec & - & 2 \\
\hline Suiriri suiriri (Vieillot, 1818) & Chaco Suiriri & - & $\mathrm{E}$ & $\mathrm{C}$ & jan-dec & - & 1 \\
\hline $\begin{array}{l}\text { Suiriri islerorum Zimmer, } \\
\text { Whittaker \& Oren, } 2001\end{array}$ & $\begin{array}{l}\text { Chapada } \\
\text { Flycatcher }\end{array}$ & - & $\mathrm{E}$ & $\mathrm{C}$ & mar-jan & - & 2 \\
\hline $\begin{array}{l}\text { Phaeomyias murina } \\
\text { (Spix, 1825) }\end{array}$ & $\begin{array}{l}\text { Mouse-coloured } \\
\text { Tyrannulet }\end{array}$ & - & $\mathrm{E}$ & $\mathrm{C}$ & jan-dec & oct & 7 \\
\hline $\begin{array}{l}\text { Capsiempis flaveola } \\
\text { (Lichtenstein, 1823) }\end{array}$ & $\begin{array}{c}\text { Yellow } \\
\text { Tyrannulet }\end{array}$ & - & $\mathrm{O}$ & $\mathrm{C}$ & feb & - & 1 \\
\hline $\begin{array}{l}\text { Phylloscartes roquettei } \\
\text { Snethlage, } 1928\end{array}$ & $\begin{array}{l}\text { Minas Gerais } \\
\text { Tyrannulet }\end{array}$ & ABR, AMG & $\mathrm{R}$ & M & jul-jan & - & - \\
\hline $\begin{array}{l}\text { Tolmomyias sulphurescens } \\
\text { (Spix, 1825) }\end{array}$ & $\begin{array}{l}\text { Yellow-olive } \\
\text { Flycatcher }\end{array}$ & - & $\mathrm{C}$ & M C & jan-dec & apr/oct & 10 \\
\hline $\begin{array}{l}\text { Tolmomyias flaviventris } \\
\text { (Wied, 1831) }\end{array}$ & $\begin{array}{l}\text { Yellow-breasted } \\
\text { Flycatcher }\end{array}$ & - & $\mathrm{R}$ & M C & $\begin{array}{l}\text { aug,oct, } \\
\text { dec }\end{array}$ & - & 1 \\
\hline $\begin{array}{l}\text { Myiophobus fasciatus } \\
\text { (Statius Muller, 1776) }\end{array}$ & $\begin{array}{l}\text { Bran-coloured } \\
\text { Flycatcher }\end{array}$ & - & $\mathrm{R}$ & $\mathrm{C}$ & jan-dec & - & - \\
\hline $\begin{array}{l}\text { Myiobius barbatus } \\
\text { (Gmelin, 1789) }\end{array}$ & $\begin{array}{l}\text { Sulphur-rumped } \\
\text { Flycatcher }\end{array}$ & - & $\mathrm{E}$ & M & jan-dec & feb & 4 \\
\hline $\begin{array}{l}\text { Lathrotriccus euleri } \\
\text { (Cabanis, 1868) }\end{array}$ & $\begin{array}{l}\text { Euler's } \\
\text { Flycatcher }\end{array}$ & - & $\mathrm{C}$ & M C & jan-dec & - & 6 \\
\hline
\end{tabular}


Table 1. Continued..

\begin{tabular}{|c|c|c|c|c|c|c|c|}
\hline Families and species & $\begin{array}{l}\text { Common } \\
\text { names }\end{array}$ & $\begin{array}{l}\text { Conservation } \\
\text { and endemism }\end{array}$ & Abundance & Habitat & $\begin{array}{l}\text { Month of } \\
\text { record }\end{array}$ & Reproduction & $\begin{array}{c}\text { Number } \\
\text { captured } \\
\text { birds } \\
\end{array}$ \\
\hline $\begin{array}{l}\text { Cnemotriccus fuscatus } \\
\text { (Wied, 1831) }\end{array}$ & $\begin{array}{l}\text { Fuscous } \\
\text { Flycatcher }\end{array}$ & - & $\mathrm{E}$ & M C & aug-apr & - & 8 \\
\hline $\begin{array}{l}\text { Contopus cinereus } \\
\text { (Spix, 1825) }\end{array}$ & Tropical Pewee & - & $\mathrm{C}$ & M C & jan-dec & - & 5 \\
\hline $\begin{array}{l}\text { Pyrocephalus rubinus } \\
\text { (Boddaert, 1783) }\end{array}$ & $\begin{array}{l}\text { Vermilion } \\
\text { Flycatcher }\end{array}$ & - & $\mathrm{R}$ & $\mathrm{H}$ & may-aug & - & - \\
\hline $\begin{array}{l}\text { Satrapa icterophrys } \\
\text { (Vieillot, 1818) }\end{array}$ & $\begin{array}{l}\text { Yellow-browed } \\
\text { Tyrant }\end{array}$ & - & $\mathrm{O}$ & $\mathrm{CV}$ & may & - & - \\
\hline $\begin{array}{l}\text { Xolmis cinereus } \\
\text { (Vieillot, 1816) }\end{array}$ & Grey Monjita & - & $\mathrm{C}$ & $\mathrm{CH}$ & apr-dec & - & - \\
\hline $\begin{array}{l}\text { Xolmis velatus } \\
\text { (Lichtenstein, 1823) }\end{array}$ & $\begin{array}{l}\text { White-rumped } \\
\text { Monjita }\end{array}$ & - & $\mathrm{C}$ & $\mathrm{CH}$ & oct-jul & - & - \\
\hline $\begin{array}{l}\text { Gubernetes yetapa } \\
\text { (Vieillot, 1818) }\end{array}$ & $\begin{array}{l}\text { Streamer-tailed } \\
\text { Tyrant }\end{array}$ & - & $\mathrm{O}$ & $\mathrm{CA}$ & oct & - & - \\
\hline $\begin{array}{l}\text { Fluvicola nengeta } \\
\text { (Linnaeus, 1766) }\end{array}$ & $\begin{array}{c}\text { Masked } \\
\text { Water-Tyrant }\end{array}$ & - & $\mathrm{R}$ & C V A H & $\begin{array}{l}\text { jan, may, } \\
\text { oct }\end{array}$ & - & - \\
\hline $\begin{array}{l}\text { Colonia colonus } \\
\text { (Vieillot, 1818) }\end{array}$ & $\begin{array}{l}\text { Long-tailed } \\
\text { Tyrant }\end{array}$ & - & $\mathrm{R}$ & C A & may,aug & - & - \\
\hline $\begin{array}{l}\text { Machetornis rixosa } \\
\text { (Vieillot, 1819) }\end{array}$ & Cattle Tyrant & - & $\mathrm{R}$ & A H & may-dec & - & - \\
\hline $\begin{array}{l}\text { Myiozetetes similis } \\
\text { (Spix, 1825) }\end{array}$ & $\begin{array}{l}\text { Social (Vermil- } \\
\text { ion-crowned) } \\
\text { Flycatcher }\end{array}$ & - & A & $\begin{array}{c}\text { M C V } \\
\text { A H }\end{array}$ & jan-dec & - & 1 \\
\hline $\begin{array}{l}\text { Pitangus sulphuratus } \\
\text { (Linnaeus, 1766) }\end{array}$ & Great Kiskadee & - & A & $\begin{array}{c}\text { M C V } \\
\text { A H }\end{array}$ & jan-dec & - & 1 \\
\hline $\begin{array}{l}\text { Myiodynastes maculatus } \\
\text { (Statius Muller, 1776) }\end{array}$ & $\begin{array}{l}\text { Streaked } \\
\text { Flycatcher }\end{array}$ & - & $\mathrm{E}$ & M C & aug-mar & oct & 14 \\
\hline $\begin{array}{l}\text { Megarynchus pitangua } \\
\text { (Linnaeus, 1766) }\end{array}$ & $\begin{array}{l}\text { Boat-billed } \\
\text { Flycatcher }\end{array}$ & - & A & $\begin{array}{c}\text { M C V } \\
\text { A H }\end{array}$ & jan-dec & - & 3 \\
\hline $\begin{array}{l}\text { Empidonomus varius } \\
\text { (Vieillot, 1818) }\end{array}$ & $\begin{array}{l}\text { Variegated } \\
\text { Flycatcher }\end{array}$ & - & $\mathrm{R}$ & M C & $\begin{array}{l}\text { mar, sep- } \\
\text { oct }\end{array}$ & - & - \\
\hline $\begin{array}{l}\text { Griseotyrannus } \\
\text { aurantioatrocristatus } \\
\text { (d'Orbigny \& Lafresnaye, 1837) }\end{array}$ & $\begin{array}{l}\text { Crowned Slaty } \\
\text { Flycatcher }\end{array}$ & - & $\mathrm{R}$ & $\mathrm{C}$ & feb,dec & - & - \\
\hline $\begin{array}{l}\text { Tyrannus albogularis } \\
\text { Burmeister, } 1856\end{array}$ & $\begin{array}{c}\text { White-throated } \\
\text { Kingbird }\end{array}$ & - & $\mathrm{E}$ & C V A H & oct-apr & - & - \\
\hline $\begin{array}{l}\text { Tyrannus melancholicus } \\
\text { Vieillot, } 1819\end{array}$ & $\begin{array}{l}\text { Tropical } \\
\text { Kingbird }\end{array}$ & - & A & C V A H & jan-dec & - & - \\
\hline Tyrannus savana Vieillot, 1808 & $\begin{array}{l}\text { Fork-tailed } \\
\text { Flycatcher }\end{array}$ & - & $\mathrm{E}$ & C V A H & aug-dec & oct & - \\
\hline $\begin{array}{l}\text { Sirystes sibilator } \\
\text { (Vieillot, 1818) }\end{array}$ & Sirystes & - & $\mathrm{C}$ & M C & jan-dec & - & 1 \\
\hline Casiornis rufus (Vieillot, 1816) & Rufous Casiornis & - & $\mathrm{C}$ & M C & jul-apr & oct & 17 \\
\hline $\begin{array}{l}\text { Myiarchus swainsoni } \\
\text { Cabanis \& Heine, } 1859\end{array}$ & $\begin{array}{l}\text { Swainson's } \\
\text { Flycatcher }\end{array}$ & - & $\mathrm{E}$ & M C & aug-dec & dec & 7 \\
\hline Myiarchus ferox (Gmelin, 1789) & $\begin{array}{l}\text { Short-crested } \\
\text { Flycatcher }\end{array}$ & - & A & M C & jan-dec & - & 14 \\
\hline $\begin{array}{l}\text { Myiarchus tyrannulus } \\
\text { (Statius Muller, 1776) }\end{array}$ & $\begin{array}{l}\text { Brown-crested } \\
\text { Flycatcher }\end{array}$ & - & $\mathrm{C}$ & M C & jan-dec & oct-nov & 19 \\
\hline \multicolumn{8}{|l|}{ PIPRIDAE (2) } \\
\hline $\begin{array}{l}\text { Neopelma pallescens } \\
\text { (Lafresnaye, 1853) }\end{array}$ & $\begin{array}{l}\text { Pale-bellied } \\
\text { Tyrant-Manakin }\end{array}$ & - & $\mathrm{R}$ & M & jul & - & 1 \\
\hline
\end{tabular}


Table 1. Continued...

\begin{tabular}{|c|c|c|c|c|c|c|c|}
\hline Families and species & $\begin{array}{l}\text { Common } \\
\text { names }\end{array}$ & $\begin{array}{l}\text { Conservation } \\
\text { and endemism }\end{array}$ & Abundance & Habitat & $\begin{array}{l}\text { Month of } \\
\text { record }\end{array}$ & Reproduction & $\begin{array}{c}\text { Number } \\
\text { captured } \\
\text { birds }\end{array}$ \\
\hline $\begin{array}{l}\text { Antilophia galeata } \\
\text { (Lichtenstein, 1823) }\end{array}$ & $\begin{array}{l}\text { Helmeted } \\
\text { Manakin }\end{array}$ & - & $\mathrm{C}$ & M C V & jan-dec & oct-dec & 7 \\
\hline \multicolumn{8}{|l|}{ TYTIRIDAE (5) } \\
\hline $\begin{array}{l}\text { Tityra inquisitor } \\
\text { (Lichtenstein, 1823) }\end{array}$ & $\begin{array}{l}\text { Black-crowned } \\
\text { Tityra }\end{array}$ & - & $\mathrm{E}$ & M C & jan-dec & - & - \\
\hline $\begin{array}{l}\text { Tityra cayana } \\
\text { (Linnaeus, 1766) }\end{array}$ & $\begin{array}{l}\text { Black-tailed } \\
\text { Tityra }\end{array}$ & - & $\mathrm{E}$ & M & oct-jun & - & - \\
\hline $\begin{array}{l}\text { Pachyramphus viridis } \\
\text { (Vieillot, 1816) }\end{array}$ & $\begin{array}{l}\text { Green-backed } \\
\text { Becard }\end{array}$ & - & $\mathrm{R}$ & M & may,oct & - & 1 \\
\hline $\begin{array}{l}\text { Pachyramphus polychopterus } \\
\text { (Vieillot, 1818) }\end{array}$ & $\begin{array}{l}\text { White-winged } \\
\text { Becard }\end{array}$ & - & $\mathrm{C}$ & M C V & jan-dec & apr/nov-dec & - \\
\hline $\begin{array}{l}\text { Pachyramphus validus } \\
\text { (Lichtenstein, 1823) }\end{array}$ & Crested Becard & - & $\mathrm{O}$ & $\mathrm{C}$ & feb & - & - \\
\hline \multicolumn{8}{|l|}{ VIREONIDAE (2) } \\
\hline $\begin{array}{l}\text { Cyclarhis gujanensis } \\
\text { (Gmelin, 1789) }\end{array}$ & $\begin{array}{l}\text { Rufous-browed } \\
\text { Peppershrike }\end{array}$ & - & A & $\begin{array}{l}\text { M C V } \\
\text { A H }\end{array}$ & jan-dec & nov & 15 \\
\hline $\begin{array}{l}\text { Vireo olivaceus } \\
\text { (Linnaeus, 1766) }\end{array}$ & Red-eyed Vireo & - & $\mathrm{R}$ & M C & feb,oct & - & 2 \\
\hline \multicolumn{8}{|l|}{ CORVIDAE (2) } \\
\hline $\begin{array}{l}\text { Cyanocorax cristatellus } \\
\text { (Temminck, 1823) }\end{array}$ & Curl-crested Jay & $\mathrm{ECe}$ & A & $\mathrm{CA} \mathrm{H}$ & jan-dec & - & - \\
\hline $\begin{array}{l}\text { Cyanocorax cyanopogon } \\
\text { (Wied, 1821) }\end{array}$ & White-naped Jay & $\mathrm{ECa}$ & $\mathrm{R}$ & $\mathrm{C}$ & feb,oct & - & - \\
\hline \multicolumn{8}{|l|}{ HIRUNDINIDAE (5) } \\
\hline $\begin{array}{l}\text { Pygochelidon cyanoleuca } \\
\text { (Vieillot, 1817) }\end{array}$ & $\begin{array}{l}\text { Blue-and-white } \\
\text { Swallow }\end{array}$ & - & $\mathrm{O}$ & $\mathrm{CV}$ & may & - & - \\
\hline $\begin{array}{l}\text { Stelgidopteryx ruficollis } \\
\text { (Vieillot, 1817) }\end{array}$ & $\begin{array}{l}\text { Southern Rough- } \\
\text { winged Swallow }\end{array}$ & - & A & C V A H & feb-nov & - & - \\
\hline $\begin{array}{l}\text { Progne tapera } \\
\text { (Vieillot, 1817) }\end{array}$ & $\begin{array}{l}\text { Brown-chested } \\
\text { Martin }\end{array}$ & - & $\mathrm{C}$ & C V A H & jan-dec & - & - \\
\hline $\begin{array}{l}\text { Tachycineta albiventer } \\
\text { (Boddaert, 1783) }\end{array}$ & $\begin{array}{l}\text { White-winged } \\
\text { Swallow }\end{array}$ & - & $\mathrm{R}$ & V A & $\begin{array}{l}\text { jan,may, } \\
\text { aug }\end{array}$ & - & - \\
\hline $\begin{array}{l}\text { Tachycineta leucorrhoa } \\
\text { (Vieillot, 1817) }\end{array}$ & $\begin{array}{l}\text { White-rumped } \\
\text { Swallow }\end{array}$ & - & $\mathrm{R}$ & V A & jul-oct & - & - \\
\hline \multicolumn{8}{|l|}{ TROGLODYTIDAE (3) } \\
\hline $\begin{array}{l}\text { Troglodytes musculus } \\
\text { Naumann, } 1823\end{array}$ & House Wren & - & A & $\mathrm{CH}$ & jan-dec & - & 2 \\
\hline $\begin{array}{l}\text { Cantorchilus leucotis } \\
\text { (Lafresnaye, 1845) }\end{array}$ & $\begin{array}{l}\text { Buff-breasted } \\
\text { Wren }\end{array}$ & - & $\mathrm{C}$ & M A & jan-dec & - & 3 \\
\hline $\begin{array}{l}\text { Donacobius atricapilla } \\
\text { (Linnaeus, 1766) }\end{array}$ & $\begin{array}{l}\text { Black-capped } \\
\text { Mockingthrush }\end{array}$ & - & $\mathrm{R}$ & $\mathrm{CV}$ & jan-dec & - & 1 \\
\hline \multicolumn{8}{|l|}{ MUSCICAPIDAE (4) } \\
\hline $\begin{array}{l}\text { Polioptila dumicola } \\
\text { (Vieillot, 1817) }\end{array}$ & $\begin{array}{c}\text { Masked } \\
\text { Gnatcatcher }\end{array}$ & - & $\mathrm{C}$ & M C & jan-dec & - & - \\
\hline $\begin{array}{l}\text { Turdus rufiventris } \\
\text { Vieillot, } 1818\end{array}$ & $\begin{array}{l}\text { Rufous-bellied } \\
\text { Thrush }\end{array}$ & - & $\mathrm{E}$ & M V A H & may-feb & - & 2 \\
\hline $\begin{array}{l}\text { Turdus leucomelas } \\
\text { Vieillot, } 1818\end{array}$ & $\begin{array}{l}\text { Pale-breasted } \\
\text { Thrush }\end{array}$ & - & $\mathrm{A}$ & M C V A & jan-dec & oct-dec & 25 \\
\hline $\begin{array}{l}\text { Turdus amaurochalinus } \\
\text { Cabanis, } 1850\end{array}$ & $\begin{array}{l}\text { Creamy-bellied } \\
\text { Thrush }\end{array}$ & - & $\mathrm{E}$ & M C V & jul-mar & - & 2 \\
\hline \multicolumn{8}{|l|}{ MIMIDAE (1) } \\
\hline $\begin{array}{l}\text { Mimus saturninus } \\
\text { (Lichtenstein, 1823) }\end{array}$ & $\begin{array}{l}\text { Chalk-browed } \\
\text { Mockingbird }\end{array}$ & & A & C V A H & jan-dec & - & - \\
\hline
\end{tabular}


Table 1. Continued..

\begin{tabular}{|c|c|c|c|c|c|c|c|}
\hline Families and species & $\begin{array}{l}\text { Common } \\
\text { names }\end{array}$ & $\begin{array}{c}\text { Conservation } \\
\text { and endemism }\end{array}$ & Abundance & Habitat & $\begin{array}{l}\text { Month of } \\
\text { record }\end{array}$ & Reproduction & $\begin{array}{c}\text { Number } \\
\text { captured } \\
\text { birds }\end{array}$ \\
\hline \multicolumn{8}{|l|}{ MOTACILLIDAE (1) } \\
\hline $\begin{array}{l}\text { Anthus lutescens } \\
\text { Pucheran, } 1855\end{array}$ & Yellowish Pipit & & $\mathrm{O}$ & C A & aug & - & - \\
\hline \multicolumn{8}{|l|}{ COEREBIDAE (1) } \\
\hline $\begin{array}{l}\text { Coereba flaveola } \\
\text { (Linnaeus, 1758) }\end{array}$ & Bananaquit & & $\mathrm{C}$ & M C & jan-dec & - & 2 \\
\hline \multicolumn{8}{|l|}{ THRAUPIDAE (15) } \\
\hline $\begin{array}{l}\text { Schistochlamys melanopis } \\
\text { (Latham, 1790) }\end{array}$ & $\begin{array}{l}\text { Black-faced } \\
\text { Tanager }\end{array}$ & & $\mathrm{O}$ & V & oct & - & - \\
\hline $\begin{array}{l}\text { Neothraupis fasciata } \\
\text { (Lichtenstein, 1823) }\end{array}$ & $\begin{array}{l}\text { White-banded } \\
\text { Tanager }\end{array}$ & $\mathrm{ECe}$ & $\mathrm{E}$ & $\mathrm{C}$ & mar-jul & - & - \\
\hline $\begin{array}{l}\text { Nemosia pileata } \\
\text { (Boddaert, 1783) }\end{array}$ & Hooded Tanager & & A & M C & jan-dec & - & - \\
\hline $\begin{array}{l}\text { Thlypopsis sordida } \\
\text { (d'Orbigny \& Lafresnaye, 1837) }\end{array}$ & $\begin{array}{l}\text { Orange-headed } \\
\text { Tanager }\end{array}$ & & $\mathrm{R}$ & M & jul & - & - \\
\hline $\begin{array}{l}\text { Cypsnagra hirundinacea } \\
\text { (Lesson, 1831) }\end{array}$ & $\begin{array}{l}\text { White-rumped } \\
\text { Tanager }\end{array}$ & & $\mathrm{R}$ & $\mathrm{C}$ & may-jan & - & - \\
\hline $\begin{array}{l}\text { Piranga flava } \\
\text { (Vieillot, 1822) }\end{array}$ & $\begin{array}{c}\text { Lowland } \\
\text { Hepatic-Tanager }\end{array}$ & & $\mathrm{E}$ & $\mathrm{CH}$ & $\begin{array}{c}\text { feb- } \\
\text { may,aug }\end{array}$ & - & - \\
\hline $\begin{array}{l}\text { Eucometis penicillata } \\
\text { (Spix, 1825) }\end{array}$ & $\begin{array}{l}\text { Grey-headed } \\
\text { Tanager }\end{array}$ & & $\mathrm{C}$ & M V & jan-dec & oct-dec & 10 \\
\hline $\begin{array}{l}\text { Tachyphonus rufus } \\
\text { (Boddaert, 1783) }\end{array}$ & $\begin{array}{l}\text { White-lined } \\
\text { Tanager }\end{array}$ & & A & M C V & jan-dec & nov-dec & 6 \\
\hline $\begin{array}{l}\text { Ramphocelus carbo } \\
\text { (Pallas, 1764) }\end{array}$ & $\begin{array}{l}\text { Silver-beaked } \\
\text { Tanager }\end{array}$ & & $\mathrm{E}$ & M C V & jan-dec & oct & 3 \\
\hline $\begin{array}{l}\text { Thraupis sayaca } \\
\text { (Linnaeus, 1766) }\end{array}$ & Sayaca Tanager & & A & $\begin{array}{l}\text { M C V } \\
\text { A H }\end{array}$ & jan-dec & oct-dec & 9 \\
\hline $\begin{array}{l}\text { Thraupis palmarum } \\
\text { (Wied, 1823) }\end{array}$ & Palm Tanager & & $\mathrm{C}$ & $\begin{array}{l}\text { M C V } \\
\text { A H }\end{array}$ & jan-dec & nov & 2 \\
\hline $\begin{array}{l}\text { Tangara cayana } \\
\text { (Linnaeus, } 1766 \text { ) }\end{array}$ & $\begin{array}{l}\text { Burnished-buff } \\
\text { Tanager }\end{array}$ & & A & $\begin{array}{l}\text { M C V } \\
\text { A H }\end{array}$ & jan-dec & - & 5 \\
\hline $\begin{array}{l}\text { Dacnis cayana } \\
\text { (Linnaeus, 1766) }\end{array}$ & Blue Dacnis & & A & M C V & jan-dec & oct & 7 \\
\hline $\begin{array}{l}\text { Hemithraupis guira } \\
\text { (Linnaeus, 1766) }\end{array}$ & Guira Tanager & & A & M C & jan-dec & nov & 7 \\
\hline $\begin{array}{l}\text { Conirostrum speciosum } \\
\text { (Temminck, 1824) }\end{array}$ & $\begin{array}{l}\text { Chestnut-vented } \\
\text { Conebill }\end{array}$ & & $\mathrm{C}$ & M C & jan-dec & - & 2 \\
\hline \multicolumn{8}{|l|}{ EMBEREZIDAE (10) } \\
\hline $\begin{array}{l}\text { Zonotrichia capensis } \\
\text { (Statius Muller, 1776) }\end{array}$ & $\begin{array}{l}\text { Rufous-collared } \\
\text { Sparrow }\end{array}$ & & A & $\mathrm{CH}$ & jan-dec & - & 1 \\
\hline $\begin{array}{l}\text { Ammodramus humeralis } \\
\text { (Bosc, 1792) }\end{array}$ & $\begin{array}{l}\text { Grassland } \\
\text { Sparrow }\end{array}$ & & $\mathrm{C}$ & $\mathrm{C}$ & jan-dec & - & - \\
\hline $\begin{array}{l}\text { Emberizoides herbicola } \\
\text { (Vieillot, 1817) }\end{array}$ & $\begin{array}{l}\text { Wedge-tailed } \\
\text { Grass-Finch }\end{array}$ & & $\mathrm{R}$ & $\mathrm{CH}$ & jan-dec & - & - \\
\hline $\begin{array}{l}\text { Volatinia jacarina } \\
\text { (Linnaeus, 1766) }\end{array}$ & $\begin{array}{l}\text { Blue-black } \\
\text { Grassquit }\end{array}$ & & $\mathrm{C}$ & $\mathrm{MC}$ & jan-dec & - & 5 \\
\hline $\begin{array}{l}\text { Sporophila plumbea } \\
\text { (Wied, 1830) }\end{array}$ & $\begin{array}{l}\text { Plumbeous } \\
\text { Seedeater }\end{array}$ & & $\mathrm{O}$ & $\mathrm{C}$ & oct & - & - \\
\hline $\begin{array}{l}\text { Sporophila lineola } \\
\text { (Linnaeus, 1758) }\end{array}$ & Lined Seedeater & & $\mathrm{O}$ & $\mathrm{C}$ & feb & - & - \\
\hline $\begin{array}{l}\text { Sporophila nigricollis } \\
\text { (Vieillot, 1823) }\end{array}$ & $\begin{array}{l}\text { Yellow-bellied } \\
\text { Seedeater }\end{array}$ & - & $\mathrm{R}$ & $\mathrm{CH}$ & feb,nov & - & \\
\hline
\end{tabular}


Table 1. Continued...

\begin{tabular}{|c|c|c|c|c|c|c|c|}
\hline Families and species & $\begin{array}{l}\text { Common } \\
\text { names }\end{array}$ & $\begin{array}{c}\text { Conservation } \\
\text { and endemism }\end{array}$ & Abundance & Habitat & $\begin{array}{l}\text { Month of } \\
\text { record }\end{array}$ & Reproduction & $\begin{array}{c}\text { Number } \\
\text { captured } \\
\text { birds } \\
\end{array}$ \\
\hline $\begin{array}{l}\text { Sporophila angolensis } \\
\text { (Linnaeus, 1766) }\end{array}$ & $\begin{array}{l}\text { Lesser (Chestnut- } \\
\text { bellied) } \\
\text { Seed-Finch }\end{array}$ & AMG & $\mathrm{O}$ & $\mathrm{CV}$ & dec & - & 1 \\
\hline $\begin{array}{l}\text { Charitospiza eucosma } \\
\text { Oberholser, } 1905\end{array}$ & $\begin{array}{l}\text { Coal-crested } \\
\text { Finch }\end{array}$ & $\mathrm{ECe}$ & $\mathrm{C}$ & $\mathrm{CH}$ & $\begin{array}{l}\text { feb- } \\
\text { mar,jul/ } \\
\text { oct }\end{array}$ & - & - \\
\hline $\begin{array}{l}\text { Coryphospingus pileatus } \\
\text { (Wied, 1821) }\end{array}$ & $\begin{array}{c}\text { Grey } \\
\text { Pileated-Finch }\end{array}$ & - & $\mathrm{C}$ & $\mathrm{CH}$ & jan-dec & - & 8 \\
\hline \multicolumn{8}{|l|}{ CARDINALIDAE (3) } \\
\hline $\begin{array}{l}\text { Saltator coerulescens } \\
\text { Vieillot, } 1817\end{array}$ & Greyish Saltator & - & $\mathrm{C}$ & M C V & jan-dec & - & - \\
\hline $\begin{array}{l}\text { Saltator similis } \\
\text { d'Orbigny \& Lafresnaye, } 1837\end{array}$ & $\begin{array}{l}\text { Green-winged } \\
\text { Saltator }\end{array}$ & - & $\mathrm{C}$ & M C & jan-dec & - & 1 \\
\hline $\begin{array}{l}\text { Saltator atricollis } \\
\text { Vieillot, } 1817\end{array}$ & $\begin{array}{l}\text { Black-throated } \\
\text { Saltator }\end{array}$ & $\mathrm{ECe}$ & A & $\mathrm{CH}$ & jan-dec & - & 1 \\
\hline \multicolumn{8}{|l|}{ PARULIDAE (4) } \\
\hline $\begin{array}{l}\text { Parula pitiayumi } \\
\text { (Vieillot, 1817) }\end{array}$ & Tropical Parula & - & $\mathrm{E}$ & M C & jan-dec & - & 1 \\
\hline $\begin{array}{l}\text { Geothlypis aequinoctialis } \\
\text { (Gmelin, 1789) }\end{array}$ & $\begin{array}{c}\text { Masked } \\
\text { Yellowthroat }\end{array}$ & - & $\mathrm{R}$ & $\mathrm{CV}$ & aug,oct & - & - \\
\hline $\begin{array}{l}\text { Basileuterus hypoleucus } \\
\text { Bonaparte, } 1830\end{array}$ & $\begin{array}{l}\text { White-bellied } \\
\text { Warbler }\end{array}$ & - & A & M & jan-dec & - & 13 \\
\hline \multicolumn{8}{|l|}{ ICTERIDAE (8) } \\
\hline $\begin{array}{l}\text { Basileuterus flaveolus } \\
\text { (Baird, 1865) }\end{array}$ & $\begin{array}{l}\text { Flavescent } \\
\text { Warbler }\end{array}$ & - & A & M C & jan-dec & dec & 25 \\
\hline $\begin{array}{l}\text { Psarocolius decumanus } \\
\text { (Pallas, 1769) }\end{array}$ & $\begin{array}{c}\text { Crested } \\
\text { Oropendola }\end{array}$ & - & A & M C V & jan-dec & - & - \\
\hline $\begin{array}{l}\text { Procacicus solitarius } \\
\text { (Vieillot, 1816) }\end{array}$ & $\begin{array}{l}\text { Solitary (Solitary } \\
\text { Black) Cacique }\end{array}$ & - & $\mathrm{R}$ & M A & jan-dec & - & - \\
\hline $\begin{array}{l}\text { Cacicus haemorrhous } \\
\text { (Linnaeus, 1766) }\end{array}$ & $\begin{array}{l}\text { Red-rumped } \\
\text { Cacique }\end{array}$ & - & $\mathrm{C}$ & M V A & jan-dec & - & - \\
\hline $\begin{array}{l}\text { Icterus cayanensis } \\
\text { (Linnaeus, 1766) }\end{array}$ & Epaulet Oriole & - & $\mathrm{E}$ & M V & jan-dec & - & - \\
\hline $\begin{array}{l}\text { Icterus jamacaii } \\
\text { (Gmelin, 1788) }\end{array}$ & Troupial & $\mathrm{ECa}$ & A & $\mathrm{CV}$ & jan-dec & - & - \\
\hline $\begin{array}{l}\text { Gnorimopsar chopi } \\
\text { (Vieillot, 1819) }\end{array}$ & Chopi Blackbird & - & A & $\mathrm{C} \mathrm{V} \mathrm{A} \mathrm{H}$ & jan-dec & oct & 3 \\
\hline $\begin{array}{l}\text { Molothrus bonariensis } \\
\text { (Gmelin, 1789) }\end{array}$ & Shiny Cowbird & - & $\mathrm{R}$ & $\mathrm{H}$ & nov-feb & - & 3 \\
\hline $\begin{array}{l}\text { Sturnella superciliaris } \\
\text { (Bonaparte, 1850) }\end{array}$ & $\begin{array}{l}\text { White-browed } \\
\text { Blackbird }\end{array}$ & - & $\mathrm{R}$ & $\mathrm{C} \mathrm{A} \mathrm{H}$ & dec-apr & - & - \\
\hline \multicolumn{8}{|l|}{ FRINGILLIDAE (1) } \\
\hline $\begin{array}{l}\text { Euphonia chlorotica } \\
\text { (Linnaeus, 1766) }\end{array}$ & $\begin{array}{l}\text { Purple-throated } \\
\text { Euphonia }\end{array}$ & - & A & M C & jan-dec & may & 15 \\
\hline
\end{tabular}

Most species were recorded in just one (71) or two (93) habitat types, while the rest were found in three (47), four (42) and five (20) habitat types (Figure 1, Table 1). The highest percent of habitat specialists was found in aquatic habitats $(16.1 \%)$, followed by the gallery forests (14.6\%) and the Cerrado vegetation (14.1\%). Only $2.7 \%$ of the recorded birds were exclusive to palm groves.

\section{Important records}

1. Greater Rhea Rhea Americana (Linnaeus, 1758)

Considered threatened in the state of Minas Gerais (Machado et al. 1998). We observed several groups of adult males and young from October to December in all sampled years. Brood size ranged from five to 17 birds. 


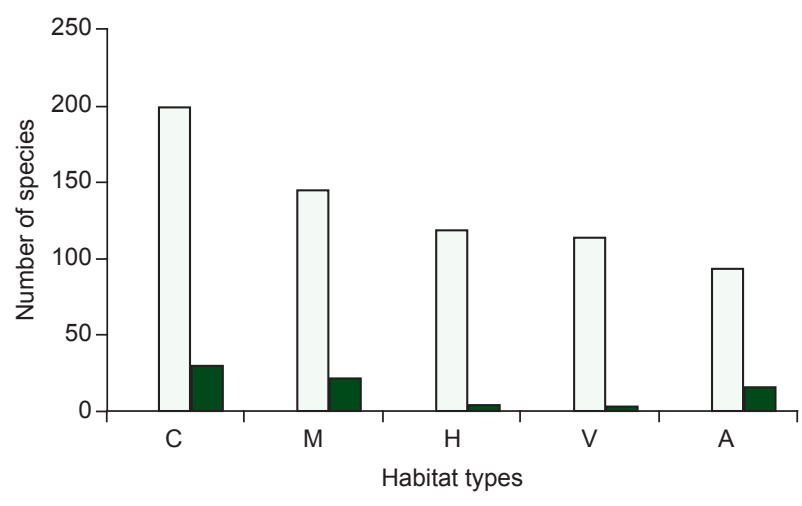

Figure 1. Occurrence of bird species per habitat in Fazenda Brejão, southeastern Brazil. C (woodlands), M (gallery forests), H (human settlements), V (palm grove swamps), A (aquatic habitats). White bars are total number of species; black bars are the exclusive species for that habitat.

Figura 1. Ocorrência de aves em cada habitat da Fazenda Brejão, sudeste do Brasil. C (cerrados), M (matas ciliares), $\mathrm{H}$ (habitações humanas), V (buritizais), A (habitats aquático). Barras brancas representam o número total de espécies; barras pretas representam o número de espécies exclusivas.

\section{Chestnut-bellied Guan Penelope ochrogaster Pelzeln, 1870}

It is a globally threatened species where population size has been estimated in fewer than 2,000 birds, split in three isolated populations in Central Brazil, Pantanal and the São Francisco River basin (Olmos 1998, Antas 2006). One solitary Chestnutbellied Guan was observed in two occasions in the gallery forest along the Paracatu River, suggesting a small population. Recent records of this species in the São Francisco basin are very scarce, and this population is considered critically endangered (Antas 2006).

\section{Bare-faced Curassow Crax fasciolata Spix, 1825}

It is considered threatened in Minas Gerais due to its ever growing rarity, mainly because of habitat loss and illegal hunting (Machado et al. 1998). There are records for the western (Marini 2001) and northwestern region of Minas Gerais (Mattos et al. 1991). At FB it was recorded only in the gallery forest along the Paracatu River, where a male was seen in October 2004 and heard April 2005 and July 2006. A pair was photographed by a camera trap in January 14, 2005 (A. Paglia unpublished data).

\section{Jabiru Jabiru mycteria (Lichtenstein, 1819)}

Although common in southwestern Brazil it is threatened in Minas Gerais. The species was first recorded in 1992, and in the following year a nest with an adult and three nestlings was recorded. This was the first documented nest of this species for the state of Minas Gerais (Encarnação \& Diniz 1988). Another nest was found in 2004, $200 \mathrm{~m}$ from the previous one, possibly belonging to the same pair, since it is known that Jabiru are very faithful to the nest site (Sick 1993). The nest was built in a large jatobá Hymenaea stigonocarpa tree, approximately $15 \mathrm{~m}$ high from the ground. Two nestlings left the nest by the end of August.

\section{Wood Stork Mycteria americana Linnaeus, 1758}

The species is threatened in the state of Minas Gerais (Rodrigues \& Michelin 2005). A nesting colony is known from the city of Vazante, about $140 \mathrm{~km}$ from FB (Encarnação \& Diniz 1998). The species occurs at FB from January to October. The highest numbers were recorded in October 2004, where 50 birds were seen in a temporary lagoon beside the Paracatu River, about $10 \mathrm{~km}$ from $\mathrm{FB}$.

6. Black-and-white Hawk-eagle Spizaetus melanoleucus (Vieillot, 1816)

It is a very rare species in Minas Gerais and considered threatened (Vasconcelos \& Veado 2001). It was recorded in two occasions in 2004, soaring over the scrubland in May and over the Paracatu River in June.

7. Blue-and-yellow Macaw Ara ararauna (Linnaeus, 1758)

Although it is a species with a wide distributional range, its population has declined in many areas mainly due to habitat loss and the illegal pet trade (BirdLife International 2000). It has been extirpated from some Brazilian states, such as Santa Catarina (Naka et al. 2002). The Blue-and-yellow Macaw is also declining in Minas Gerais, and nowadays its population has become restricted to the northwest region (Rodrigues \& Goulart 2005, Rodrigues 2008). The Blue-and-yellow Macaw is a frequent inhabitant of FB, where the palm grove swamps are its prime habitat. We have seen pairs breeding in dead Mauritia flexuosa palm trees. The palm trees are also used for overnight roosting sites. Highest numbers are recorded late October, when the fruits of 'pequi' Caryocar brasiliensis (Caryocaraceae) start to ripen. Other food resources for this species are the fruits of 'cumbaru' Dypteryx alata (Leguminosae) in March, the 'buriti' M. flexuosa in August and October, the 'pau-terrinha' Qualea parviflora, 'pau-terra' Q. grandiflora, and 'pau-doce' Vochysia cinnamomea, all in August. The availability of fruits in August coincides with the fledging of young. The Blue-and-yellow Macaw does not use communal roosting sites as observed for other parrots at FB (Carrara et al. 2007).

\section{Cactus Parakeet Aratinga cactorum (Kuhl, 1820)}

It is considered endemic to the Caatinga dry lands of northeastern Brazil (Sick 1993, Stotz et al. 1996). It is a common species in the tall scrublands of FB, and it was recorded throughout all months of the year. It formed mixed species flocks together with the Peach-fronted Parakeet Arating a aurea. The Cactus Parakeet was recorded eating fruits of 'aroeira' Myracrodruon urundeuva (Anacardiaceae) in the gallery forests of the Paracatu River in August.

\section{Yellow-faced Parrot Alipiopsitta xanthops (Spix, 1824)}

It is considered endemic to the Cerrado region (Silva \& Bates 2002) and threatened in Minas Gerais due to habitat loss and the illegal pet trade (Machado et al. 1998). It is a common species at $\mathrm{FB}$, recorded throughout the year, usually seen in flocks of 10 to 15 birds. A flock of approximately 50 birds were recorded daily in March 2002 at a fig tree (Ficus sp. Moraceae) located at the FB headquarters. This flock was also recorded eating fruits of 'jaboticaba' Myrciaria cauliflora, guava Psidium guajava (both Myrtaceae), and unripe fruits of mango Mangifera indica (Anacardiaceae). The Yellow-faced Parrot use Eucalyptus stands for overnight roosting together with the Blue-fronted Parrot Amazona aestiva. At least 160 birds using this roosting site were counted (Carrara et al. 2007).

10. Black Jacobin Florisuga fusca (Vieillot, 1817)

It is a typical hummingbird species of the Atlantic forest of eastern Brazil, even considered endemic to that biome (Stotz et al. 1996). The Black Jacobin was recorded twice at FB: July 2003 in a scrubland area and in August 2003 flying over the gallery 
forest of the Paracatu River. This and previous records (Bagno \& Marinho-Filho 2001) demonstrate that the range of this species extends a fair distance beyond the Atlantic Forest borders, indicating that its endemic status must be revaluated.

\section{Suiriri Flycatcher Suiriri suriri (Vieillot, 1818)}

This species has a complex taxonomy, and three forms are acknowledged: S. s. suiriri, S. s. affinis e S. s. bahiae (Fitzpatrick 2004). The nominal form occurs mainly in the Chaco, from central Bolivia, Paraguay, northern Argentina to southern Brazil and Uruguay. S. s. affinis is widely distributed over the Brazilian Cerrado and eastern Bolivia, but it is also found in savanna habitats in Amazon forest in northern Brazil and southern Suriname (Hayes 2001, Zimmer et al. 2001, Fitzpatrick 2004). S. s. bahiae, found in the dry Caatinga of northeastern Brazil, is not a consensual form, and may be a hybrid of affinis and suiriri (Hayes 2001, Zimmer et al. 2001, Fitzpatrick 2004). S. s. affinis is a common species at $\mathrm{FB}$, where it can be observed in pairs or small groups as described by Lopes \& Marini (2005 and 2006). In FB we also found a few individuals bearing the nominal form characteristics, such as a smaller body size, whitish underparts, grayish rump, and entirely dark tail feathers. Two of these birds that were tape recorded and collected were associated in the same flock, with two other birds with affinis characteristics, which were also collected. It is important to note that FB is the only site in central and northeastern Brazil where white-bellied birds were recently observed. The few records of white-bellied individuals for northeastern Brazil are old museum skins, sometimes considered white-bellied bahiae or authentic members of the nominal form (Pinto \& Camargo 1961, Hayes 2001).

12. Minas Gerais Tyrannulet Phylloscartes roquettei Snethlage, 1928

The Minas Gerais Tyrannulet was until the 1990's known only from the type locality, in northern Minas Gerais, but was subsequently found in several new localities (Raposo et al. 2002, Luiz et al. 2006, Vasconcelos et al. 2006). It is considered a critically endangered species (BirdLife International 2000). Two individuals were observed on 24 July 2006 in the riparian forests of the Paracatu River ( $17^{\circ} 04^{\prime} 26^{\prime \prime} \mathrm{S}$ and $45^{\circ} 54^{\prime} 13$ ' W). On the following day, two additional individuals were observed foraging some $200 \mathrm{~m}$ from this site. This riparian forest is $15-20 \mathrm{~m}$ tall and semi-deciduous, with some areas covered by terrestrial bromeliads (gravatás). This and other unpublished records of the Minas Gerais Tyrannulet are best described in Lopes et al. (2008).

\section{Discussion}

The survey revealed high bird species richness for the FB. Moreover, the region harbors threatened, rare and endemic species which reinforces the high conservation relevance of the area for the Cerrado biome.

The number of species at FB corresponds to $32 \%$ of all species to date recorded for the entire Cerrado region of South America (Silva 1995b). Avian diversity in the FB area is as high as that documented for some already established protected areas of Brazilian Cerrado: 'Serra do Cipó' national park (226 species, Rodrigues et al. 2005); 'Serra da Canastra' national park (282 species, Silveira 1998); 'Águas Emendadas' biological reserve (300 species, Bagno 1998, Lopes et al. 2005).

Sixteen species recorded between 1991 and 1994 by two of us (CDE and MGD) were not recorded in the present survey. Among those there are aquatic species such as the two grebes Tachybaptus dominicus (Linnaeus, 1766), Podilymbus podiceps (Linnaeus, 1758), and the Masked Duck Nomonyx dominica. Some species need voucher confirmation because it would represent large range extensions such as Patagioenas plumbea (Vieillot, 1818), Chordeiles acutipennis (Hermann, 1783), Amazilia lactea (Lesson, 1832), Picumnus cirratus Temminck, 1825, and Serpophaga subcristata (Vieillot, 1817). Sicalis citrina Pelzeln, 1870 occurs mainly at high elevations and also would need a voucher confirmation. Other species are of probable occurrence in the area, and some of them were not recorded, possibly due to their low density in the area such as the Black-collared Hawk Busarellus nigricollis (Latham, 1790), the Spotted Sandpiper Actitis macularius (Linnaeus, 1766), the White-tailed Goldenthroat Polytmus guainumbi (Pallas, 1764), the White-headed Marsh-Tyrant Arundinicola leucocephala (Linnaeus, 1764), the Gray-eyed Greenlet Hylophilus amaurocephalus (Nordmann, 1835), and the Cinnamon Tanager Schistochlamys ruficapillus (Vieillot, 1817). Also, the Red-cowled Cardinal Paroaria dominicana (Linnaeus, 1758), a typical species of the dry Caatinga biome of northeastern Brazil (Ridgely \& Tudor 1989 ) is at the edge of its distribution at FB. We also cannot disregard the hypothesis that this bird was a freed cage bird.

It is important to comment on the possible local extinction of the Red-and-green Macaw Ara chloroptera. Its original range has been diminished as a consequence of habitat loss and the illegal pet trade (Machado et al. 1998). There are records from 20 years ago for FB, the last one being of an individual paired with a Blue-andyellow Macaw.

Some species appear to use FB only as a stopover site. Members of the Scolopacidae recorded at FB breed at high latitudes in North America and winter in the southern latitudes of South America. The seasonal lagoons at FB are the preferred habitats for these species. Other species appear at FB during the austral winter (e.g. the Vermilion Flycatcher Pyrocephalus rubinus). These species arrive in northwestern Minas Gerais to escape from the cold winter of the south (Sick 1993). We did not find these species in reproductive condition at FB.

Most migrant species arrive at FB at the end of the dry season. Some birds arrive up to December (Plumbeous Kite Ictinia plumbea, Fork-tailed Flycatcher Tyrannus savana, Swainson's Flycatcher Myiarchus swainsoni); others arrive from December to April (Blue Ground-Dove Claravis pretiosa, White-browed Blackbird Sturnella superciliaris); and others occur from September to April (Picui Ground-dove Columbina picui, Lesser Elaenia Elaenia chiriquensis, Greenish Elaenia Myiopagis viridicata, Streaked Flycatcher Myiodynastes maculatus, White-throated Kingbird Tyrannus albogularis). The arrival of these species is coincident with the peak of insect and fruit availability in the Cerrado (Macedo 2002), and many of them breed in the area (as confirmed by the presence of a brood patch). Also, more than $85 \%$ of the species started breeding activities at the end of the dry season and beginning of the raining season (unpublished data), which is the usual pattern of breeding for most Cerrado species (Marini \& Durães 2001).

The high species richness of birds recorded at FB may be related to the diversity of habitats and to the size of the area. The location of FB also seems to be important. The Paracatu River still holds large areas of gallery forests, which support a high number of forestdependent species (Rodrigues \& Faria 2007). The seasonal flooding of this river is an important event for the aquatic birds. This event is of vital importance for fish reproduction, a prime food item for many storks, herons and grebes. The storks seem to depend on this water cycle for reproduction (Sick 1993). Thus, aquatic habitats and the gallery forests, although less representative in terms of area within FB compared to the woodlands, held at least 18 exclusive species.

Among the most important habitats at FB, the palm groves are of prime importance for many birds, despite having few exclusive species. The palm groves occur along flat areas near springs in swampy grounds or in dense stands along the gallery forest. This habitat, once 
common in the northwest of Minas Gerais state, is now becoming rare (Goulart 2005). The palm grove swamps serve as an important role in the maintenance of the bird diversity of FB, serving as overnight roosting sites, as a source of food (its fruits) and water, and as a site for reproduction for many bird species (see also (Carvalho 1991, Sick 1993). Almost $42 \%$ of the bird species at FB were recorded in the palm groves. Some of these birds are closely tied to the palm groves (e.g., the Blue-and-yellow Macaw, Red-bellied Macaw Orthopsittaca manilata, and Fork-tailed Palm-swift Tachornis squamata, all of them use the palms as nesting sites). Many other birds such as parrots (11 species) also use the palm fruit as a food item. Two passerine birds, Large-billed Antwren Herpsilochmus longirostris and Stripe-necked Tody-tyrant Hemitriccus striaticollis, appear to be restricted to the palm groves.

FB is also important for large species which need large home ranges such as the Greater Rhea Rhea americana, Horned Screamer Anhima cornuta, Blue-and-Yellow Macaw, parrots and many raptorial species.

The Cerrado region of central Brazil is rapidly being replaced with crops and pasture. Over the past 35 years, more than half of the Cerrado's original area of two million square kilometers has been converted to agriculture (Machado et al. 2004b). It is now among the world's top regions for the production of beef and soybeans (WWF 1995). Just $2.2 \%$ of the Cerrado is protected, and it is losing ground faster than the Amazon rainforest to the north. At the current rate of loss, this ecosystem could be gone by 2030 (Machado et al. 2004b). This reality is not different for the northwest of Minas Gerais, a vast region of the industrialized southeastern Brazil yet to be 'developed'. The lands surrounding FB are already in the process of transformation, and one can predict that FB will become an 'island' of native vegetation.

Within FB, one of the main threats is the overgrazing of the understory by cattle that invade the area. This is because FB is not completely surrounded by fences. Thus, cattle are practically allowed free year-round access to the area, a practice that has resulted in trampling of soil and vegetation, destruction of the river banks, and lack of tree regeneration (including the palm trees). Illegal hunting conducted by local neighbors is also an important threat yet to be evaluated. We recorded destruction of Macaw nests built in palm trees by poachers several times. Possibly, this has the potential to reduce the population viability of the Macaws in the area.

The present work is one of the rare faunal surveys ever done for northwestern Minas Gerais (see also Mattos et al. 1991). Many researchers have called attention to this region, which is considered a priority area for biological conservation (Drummond et al. 2005). The results showed here support for the importance of palm grove habitats for the maintenance of bird diversity, a habitat that has been overlooked by Brazilian authorities since the time of the establishment of protected areas. Northwestern Minas Gerais lacks federal large reserves in this important region (Camargos 2001).

\section{Acknowledgements}

This work was supported by 'Vallourec \& Mannesmann Florestal S.A.' (V\&M). We are very grateful to the staff of V\&M, specially G.D. de Freitas, M.A. Moura and A. Claret for their support in all the phases of the project. MR thanks the CNPq (Brazilian Research Agency) for a research grant (300731/2006-0) and CNPq (473428/2004-0), Fapemig (PPM CRA APQ-0434-5.03/07) and Fundação O Boticário de Proteção à Natureza for supporting the 'Laboratório de Ornitologia' of 'Universidade Federal de Minas Gerais'. LEL received a doctoral fellowship from FAPEMIG during this study. We thank IBAMA and ICMbio/CEMAVE for the ringing and collection permits.

\section{References}

AB'SABER, A.N. 1977. Os domínios morfoclimáticos na América do Sul, primeira aproximação. Geomorfologia. 52(1):1-21.

AMERICAS, W.F.T. 1993. Censo neotropical de aves aquáticas 1992: Brasil. IBAMA/Cemave, Brasília, p.38.

ANTAS, P.T.Z. 2006. Chestnut-bellied Guan (Penelope ochrogaster). In Conserving cracids: the most threatened family of birds in the americas (D.M. Brooks, ed.). Miscellaneous Publications of the Houston Museum of Natural Science, Houston, p. 75-78. (n. 6).

BAGNO, M.A. 1998. As aves da Estação Ecológica de Águas Emendadas. In Vertebrados da Estação Ecológica de Águas Emendadas, história natural e ecologia em um fragmento de Cerrado do Brasil central (J. MarinhoFilho, F. Rodrigues \& M. Guimarães, eds.). SEMATEC/IEMA/IBAMA, Brasília, p. 22-33.

BAGNO, M.A. \& MARINHO-FILHO, J. 2001. A avifauna do Distrito Federal: uso de ambientes abertos e florestais e ameaças. In Cerrado: caracterização e recuperação de matas de galeria (J.F. Ribeiro, C.E.L. Fonseca \& J.C. Sousa-Silva, eds.). Embrapa Cerrados, Planaltina, p. 495-528.

BirdLife International. 2000. Threatened birds of the World. Lynx, Barcelona, p.852.

CAMARGOS, R.M.F. 2001. Unidades de conservação em Minas Gerais: levantamento e discussão. Publicações Avulsas da Fundação Biodiversitas, Belo Horizonte, p. 7-67. (n. 2).

CARRARA, L.A., FARIA, L.P., AMARAL, F.Q. \& RODRIGUES, M. 2007. Dormitórios do papagaio-verdadeiro Amazona aestiva e do papagaiogalego Salvatoria xanthops em plantio comercial de eucalipto. Revista Brasileira de Ornitologia. 15(1):135-138.

CARVALHO, P.G.S. 1991. As veredas e sua importância no domínio dos cerrados. Informe Agropecuário. 15(168):54-56.

Comitê Brasileiro de Registros Ornitológicos - CBRO. 2008. Lista das aves do Brasil. CBRO. Available at: http://www.ib.usp.br/cbro. [Accessed in 20/02/2009].

COLLAR, N.J., GONZAGA, L.P., KRABBE, N., MADROÑO NIETO, A., NARANJO, L.G., PARKER III, T.A. \& WEGE, D.C. 1992. Threatened birds of the America: the ICBP/IUCN Red Data Book. International Council for Bird Preservation, Cambridge.

DRUMMOND, G.M., MARTINS, C.S., MACHADO, Â.B.M., SEBAIO, F.A. \& ANTONINI, Y. 2005. Biodiversidade em Minas Gerais: um atlas para sua conservação. Fundação Biodiversitas, Belo Horizonte, p.222.

EITEN, G. 1972. The cerrado vegetation of Brazil. Bot. rev.. 38(1):201-341.

ENCARNAÇÃO, C.D. \& DINIZ, M.G. 1988. Jabiru mycteria (Lichtenstein, 1819). In Livro vermelho das espécies ameaçadas de extinção da fauna do Estado de Minas Gerais (A.B.M. Machado, G.A.B. Fonseca, R.B. Machado, L.M.S. Aguiar \& L.V. Lins, eds.). Fundação Biodiversitas, Belo Horizonte, p. 195-197.

ENCARNAÇÃO, C.D. \& DINIZ, M.G. 1998. Mycteria americana Linnaeus, 1758. In Livro vermelho das espécies ameaçadas de extinção da fauna do Estado de Minas Gerais (A.B.M. Machado, G.A.B. Fonseca, R.B. Machado, L.M.S. Aguiar \& L.V. Lins, eds.). Fundação Biodiversitas, Belo Horizonte, p. 198-200.

FITZPATRICK, J. 2004. Family Tyrannidae (Tyrant-flycatchers). In Handbook of the birds of the world (J. Hoyo, A. Elliott \& D. Christie, eds.). Lynx, Barcelona, p. 170-462. (v. 9, Cotingas to pipits and wagtails).

GOULART, E.M.A. 2005. Navegando o Rio das Velhas das Minas aos Gerais. Instituto Guaicuy-SOS Rio das Velhas, Belo Horizonte, p.754.

HAYES, F.E. 2001. Geographical variation, hybridization, and the leapfrog pattern of evolution in the Suiriri Flycatcher (Suiriri suiriri) complex. Auk. 118(2):457-471.

HILTY, S.L. \& BROWN, W.L. 1986. A guide to the birds of Colombia. Princeton University Press, New Jersey, p. 512.

LOPES, L.E., LEITE, L., PINHO, J.B. \& GOES, R. 2005. New bird records to the Estação ecológica de Águas Emendadas, Planaltina, Distrito Federal. Ararajuba. 13(2):107-108. 
LOPES, L.E., MALDONADO-COELHO, M., HOFFMANN, D., LUIZ, E.R. \& D'ANGELO NETO, S. 2008. Geographic distribution, habitat association, and conservation status of the Critically Endangered Minas Gerais Tyrannulet Phylloscartes roquettei. Bird Cons. Int. 18(1):53-62.

LOPES, L.E. \& MARINI, M.Â. 2005. Biologia reprodutiva de Suiriri affinis e S. islerorum (Aves: Tyrannidae) no Cerrado do Brasil Central. Papéis Avulsos de Zoologia. 45:127-141.

LOPES, L.E. \& MARINI, M.Â. 2006. Home range and habitat use by Campo Suiriri (Suiriri affinis) and Chapada Flycatcher (Suiriri islerorum) in the Central Brazilian Cerrado. St. Neot. Fauna Envir.. 41(1):87-92.

LUIZ, E.R., RIBON, R., MATTOS, G.T. \& MORAES, L.L. 2006. Discovery of Minas Gerais Tyrannulet Phylloscartes roquettei in the Jequitinhonha Valley, north-east Minas Gerais, Brazil. Cotinga. 26(1):84-86.

MACEDO, R.H.F. 2002. The avifauna: ecology, biogeography, and behavior. In The cerrados of Brazil: ecology and natural history of a neotropical savanna (P.S. Oliveira \& R.J. Marquis, eds.). Columbia University Press, New York, p. 242-265.

MACHADO, A.B.M., FONSECA, G.A.B., MACHADO, R.B., AGUIAR, L.M.S. \& LINS, L.V. 1998. Livro vermelho das espécies ameaçadas de extinção da fauna de Minas Gerais. Biodiversitas, Belo Horizonte, p.605.

MACHADO, R.B., RAMOS-NETO, M.B., HARRIS, M.B., LOURIVAL, R. \& AGUIAR, L.M.S. 2004a. Análise de lacunas de proteção da biodiversidade no Cerrado: Brasil. In Congresso Brasileiro de Unidades de Conservação. Universidade Livre do meio Ambiente, Curitiba, p. 29-38.

MACHADO, R.B., RAMOS NETO, M.B., PEREIRA, P.G.P., CALDAS, E.F., GONÇALVES, D.A., SANTOS, N.S., TABOR, K. \& STEININGER, M. 2004b. Estimativas de perda da área do Cerrado brasileiro. Conservação Internacional, Brasília, p. 32.

MARINI, M.Â. 2001. Effects of forest fragmentation on birds of the Cerrado region, Brazil. Bird Cons. Int. 11(1):13-25.

MARINI, M.Â. \& DURÃES, R.D. 2001. Annual pattern of molt and reproductive activity of Passerines in south-central Brazil. Condor. 115(3):767-775.

MARRIS, E. 2005. The forgotten ecosystem. Nature. 437:944-945.

MATTOS, G.T., ANDRADE, M.A. \& FREITAS, M.V. 1991. Levantamento de aves silvestres na região noroeste de Minas Gerais. Revista da Sociedade Ornitológica Mineira. 39(1):26-29.

MMA (Ministério do Meio Ambiente). 2003. Instrução Normativa MMA de 27 de maio de 2003. Disponível em http://www.mma.gov.br.

NAKA, L.N., RODRIGUES, M., ROOS, A.L. \& AZEVEDO, M.A. 2002. Bird conservation on the island of Santa Catarina. Bird Cons. Int. 12(2):123-150.

OLIVEIRA-FILHO, A.T. \& RATTER, J.A. 2002. Vegetation physiognomies and woody flora of the cerrado biome. In The cerrados of Brazil: ecology and natural history of a neotropical savanna (P.S. Oliveira \& R.J. Marquis, eds.). Columbia University Press, New York, p. 91-120.

OLIVEIRA, P.S. \& MARQUIS, R.J. 2002. The cerrado of Brazil: ecology and natural history of a neotropical savanna. Columbia University Press, New York, p. 326.

OLMOS, F. 1998. The Chestnut-bellied Guan (Penelope ochrogaster) in the northern Pantanal of Poconé, Matto Grosso, Brazil. Bulletin of the IUCN/ BirdLife/WPA Cracid Specialist Group. 6:7-10.

OLMOS, F. 2006. Aves ameaçadas, prioridades e políticas de conservação no Brasil. Natureza \& Conservação. 3(1):21-42.

PINTO, O.M.O. \& CAMARGO, E.A. 1961. Resultados ornitológicos de quatro recentes expedições do Departamento de Zoologia ao nordeste do Brasil, com a descrição de seis novas subespécies. Arq. zool. Est. Sao Paulo. 11:193-284.

RAPOSO, M.A., BARNETT, J.M., KIRWAN, G.M. \& PARRINI, R. 2002. New data concerning the distribution, behaviour, ecology and taxonomic relationships of Minas Gerais Tyrannulet Phylloscartes roquettei. Bird Cons. Int. 12(2):241-253.

RIDGELY, R.S. \& TUDOR, G. 1989. The birds of South America. University of Oxford Press, Oxford, p. 516. (v. 1, The oscine passerines).
RIDGELY, R.S. \& TUDOR, G. 1994. The birds of South America. University of Oxford Press, Oxford, p.811. (v. 2, The suboscine passerines).

RODRIGUES, M. 2008. Noteworthy bird records at Lagoa Santa, southeastern Brazil. Rev. Bras. Zool. 25(1):150-153.

RODRIGUES, M., CARRARA, L.A., FARIA, L.P. \& GOMES, H.B. 2005. Aves do Parque Nacional da Serra do Cipó: o vale do Rio Cipó, Minas Gerais, Brasil. Rev. Bras. Zool. 22(2):326-338.

RODRIGUES, M. \& FARIA, L.P. 2007. Species richness of understory birds in different habitats of the Cerrado Region of South-eastern Brazil. Ecotropica. 13(2):101-110.

RODRIGUES, M. \& GOULART, F.F. 2005. Aves regionais: de Burton aos dias de hoje. In Navegando o Rio das Velhas das Minas aos Gerais (E.M.A. Goulart, ed.). Instituto Guaicuy - SOS Rio das Velhas, Belo Horizonte, p. 590-603.

RODRIGUES, M. \& MICHELIN, V.B. 2005. Riqueza e diversidade de aves aquáticas de uma lagoa natural no sudeste do Brasil. Rev. Bras. Zool.. 22(4):928-935.

SANTOS, F.R., GUIMARÃES, P.E.M. \& REDONDO, R.A. 2002. Bancos de DNA: coleções estratégicas para estudos da biodiversidade. Lundiana. 3(1):93-98.

SICK, H. 1993. Birds in Brazil. Princeton University Press, Princeton, p.703.

SILVA, J.M.C. 1995a. Avian inventory of the cerrado region, South America: implications for biological conservation. Bird Cons. Int. 5(2):291-304.

SILVA, J.M.C. 1995b. Birds of the cerrado region, South America. Steenstrupia. 21(1):69-92.

SILVA, J.M.C. \& BATES, J.M. 2002. Biogeographic patterns and conservation in the South American cerrado: a tropical savanna Hotspot. Bioscience. 52(2):225-233.

SILVA, J.M.C. \& SANTOS, M.P.D. 2005. A importância relativa dos processos biogeográficos na formação da avifauna do Cerrado e de outros biomas brasileiros. In Cerrado: ecologia, biodiversidade e conservação (A. Scariot, J.C. Souza-Silva \& J.M. Felfili, eds.). Ministério do Meio Ambiente, Brasília, p. 220-233.

SILVA, J.M.C., SOUZA, M.C. \& CASTELLETI, C.H.M. 2004. Areas of endemism for passerine birds in the Atlantic forest, South America. Global ecol. biogeogr.13(1):85-92.

SILVEIRA, L.F. 1998. The birds of Serra da Canastra National Park and adjacent areas, Minas Gerais, Brazil. Cotinga. 10(2):55-63.

STOTZ, D.F., FITZPATRICK, J.W., PARKER III, T. \& MOSKOVITS, D.K. 1996. Neotropical birds: ecology and conservation. University of Chicago Press, Chicago, Illinois, p. 882.

VASCONCELOS, M.F., D'ANGELO NETO, S., KIRWAN, G.M., BORNSCHEIN, M.R., DINIZ, M.G. \& SILVA, J.F. 2006. Important ornithological records from Minas Gerais state, Brazil. Bull. Br. Ornithol. Club. 126(2):212-238.

VASCONCELOS, M.F. \& VEADO, E.M.V. 2001. Nova ocorrência do gavião-pato (Spizastur melanoleucus) no estado de Minas Gerais. Atual. ornitol. 103(1):7.

VIEIRA, L.G., PAGLIA, A.P., LOPES, W., OLIVEIRA, F., MOURA Jr., A.J. \& DINIZ, R.F. 2005. Ocorrência e abundância de Pecary tajacu registrados por armadilhas fotográficas no noroeste de Minas Gerais. Suiform Soundings. 5(1):21-23.

World Wildlife Fund - WWF. 1995. De grão em grão o cerrado perde o espaço. Cerrado: impactos do processo de ocupação. WWF, Brasília, p. 12.

ZIMMER, K.J., WHITTAKER, A. \& OREN, D.C. 2001. A cryptic new species of flycatcher (Tyrannidae: Suiriri) from the Cerrado region of central South America. Auk. 118(1):56-78. 